\title{
Article \\ Methylmercury-Induced Metabolic Alterations in Caenorhabditis elegans Are Diet-Dependent
}

\author{
Nicole Crawford ${ }^{1}$, Megan Martell ${ }^{1}$, Tyson Nielsen ${ }^{1}$, Belal Khalil ${ }^{1}$, Farooq Imtiaz ${ }^{1}$, Etienne Nguidjo ${ }^{1}$, \\ Jennifer L. Newell-Caito ${ }^{2}{ }^{(0}$, Julia Bornhorst $\left.{ }^{3}{ }^{(}\right)$, Tanja Schwerdtle ${ }^{4}$ and Samuel W. Caito ${ }^{1, *}$ \\ 1 Department of Pharmaceutical Sciences, Husson University School of Pharmacy, Bangor, ME 04401, USA; \\ crawfordn@husson.edu (N.C.); martellm@husson.edu (M.M.); nielsent@husson.edu (T.N.); \\ khalilb@husson.edu (B.K.); imtiazf@husson.edu (F.I.); etiennenguidjo08@yahoo.ca (E.N.) \\ 2 Department of Molecular and Biomedical Sciences, University of Maine, Orono, ME 04469, USA; \\ jennifer.newellcaito@maine.edu \\ 3 Food Chemistry, Faculty of Mathematics and Natural Sciences, University of Wuppertal, \\ 42119 Wuppertal, Germany; bornhorst@uni-wuppertal.de \\ 4 Department of Food Chemistry, Institute of Nutritional Science, University of Potsdam, \\ 14558 Potsdam, Germany; tanja.schwerdtle@bfr.bund.de \\ * Correspondence: caitos@husson.edu
}

\section{check for} updates

Citation: Crawford, N.; Martell, M.; Nielsen, T.; Khalil, B.; Imtiaz, F.; Nguidjo, E.; Newell-Caito, J.L.; Bornhorst, J.; Schwerdtle, T.; Caito, S.W. Methylmercury-Induced Metabolic Alterations in Caenorhabditis elegans Are Diet-Dependent. Toxics 2021, 9, 287. https://doi.org/10.3390/toxics9110287

Academic Editors: Richard Ortega and Asuncion Carmona

Received: 1 September 2021

Accepted: 29 October 2021

Published: 2 November 2021

Publisher's Note: MDPI stays neutral with regard to jurisdictional claims in published maps and institutional affiliations.

Copyright: (c) 2021 by the authors. Licensee MDPI, Basel, Switzerland. This article is an open access article distributed under the terms and conditions of the Creative Commons Attribution (CC BY) license (https:// creativecommons.org/licenses/by/ $4.0 /)$.
Abstract: Methylmercury (MeHg) is a well-known neurotoxicant; however, its role in metabolic diseases has been gaining wider attention. Chronic exposure to $\mathrm{MeHg}$ in human populations shows an association with diabetes mellitus and metabolic syndrome (MS). As the incidences of both obesity and MS are on the rise globally, it is important to understand the potential role of $\mathrm{MeHg}$ in the development of the disease. There is a dearth of information on dietary interactions between $\mathrm{MeHg}$ and lipids, which play an important role in developing MS. We have previously shown that $\mathrm{MeHg}$ increases food seeking behaviors, lipid levels, fat storage, and pro-adipogenic gene expression in $C$. elegans fed the standard OP50 Escherichia coli diet. However, we hypothesized that these metabolic changes could be prevented if the worms were fed a bacterial diet lower in lipid content. We tested whether C. elegans developed metabolic alterations in response to $\mathrm{MeHg}$ if they were fed two alternative E. coli strains (HT115 and HB101) that are known absorb significantly less lipids from their media. Additionally, to explore the effect of a high-lipid and high-cholesterol diet on $\mathrm{MeHg}$-induced metabolic dysfunction, we supplemented the OP50 strain with twice the standard concentration of cholesterol in the nematode growth media. Wild-type worms fed either the HB101 or HT115 diet were more resistant to $\mathrm{MeHg}$ than the worms fed the OP50 diet, showing a significant right-hand shift in the dose-response survival curve. Worms fed the OP50 diet supplemented with cholesterol were more sensitive to $\mathrm{MeHg}$, showing a significant left-hand shift in the dose-response survival curve. Changes in sensitivity to $\mathrm{MeHg}$ by differential diet were not due to altered $\mathrm{MeHg}$ intake in the worms as measured by inductively coupled mass spectrometry. Worms fed the low-fat diets showed protection from MeHg-induced metabolic changes, including decreased food consumption, lower triglyceride content, and lower fat storage than the worms fed either of the higher-fat diets. Oxidative stress is a common characteristic of both $\mathrm{MeHg}$ exposure and high-fat diets. Worms fed either OP50 or OP50 supplemented with cholesterol and treated with $\mathrm{MeHg}$ had significantly higher levels of reactive oxygen species, carbonylated proteins, and loss of glutathione than the worms fed the HT115 or HB101 low-lipid diets. Taken together, our data suggest a synergistic effect of $\mathrm{MeHg}$ and dietary lipid levels on $\mathrm{MeHg}$ toxicity and fat metabolism in C. elegans, which may affect the ability of $\mathrm{MeHg}$ to cause metabolic dysfunction.

Keywords: methylmercury; diet; cholesterol; high fat; low fat 


\section{Introduction}

Metabolic syndrome (MS) and obesity are major health concerns with increasing prevalence worldwide. MS is defined as a multifactorial condition characterized by insulin resistance, diabetes mellitus (DM), dyslipidemia, and obesity. It has become increasingly evident that many factors influence the prevalence of MS, including environmental factors $[1,2]$. One such environmental agent emerging as a potential obesogen is methylmercury $(\mathrm{MeHg})$. MeHg is a well-known neurotoxin, which, in developmental exposures, causes cognitive and behavioral dysfunction in children and is linked to the development of neurodegenerative diseases, such as Parkinson's disease [3,4]. While currently being debated, there is growing evidence for a link between $\mathrm{MeHg}$ exposure and the development of MS. The National Health and Nutrition Examination Survey (NHANES) and Korean NHANES (KNHANES) data from 2003-2014 and 2011-2013, respectively, support an association between blood heavy metal levels (which include Hg) with MS, obesity, and lipid dysregulation [5-7]. These studies highlight the effect of heavy metals on the development of MS; however, it is unclear as to whether the observed metabolic effects were due to a single metal or a synergism of multiple metals in the mixture. However it has been shown that elevated blood mercury levels are also associated with increased visceral adipose tissue [8], and that toenail mercury levels (a marker for chronic Hg exposure) are associated with the development of MS [9]. We have recently shown that MeHg significantly increased lipid storage, altered feeding behavior, and increased the transcription of MS-related genes in Caenorhabditis elegans (C. elegans) [10]. Mechanisms that lead to MeHg-induced dyslipidemia are not known.

As a toxicant, $\mathrm{MeHg}$ primarily enters the human body through our diet. $\mathrm{MeHg}$ is a major contaminant of our fish supply, with greater $\mathrm{Hg}$ concentrations present in fish higher up the food chain, such as tuna, shark, swordfish, and mackerel [11]. While there are many dietary benefits from regular fish consumption, such as increased polyunsaturated fatty acids (PUFA) and selenium intake, the level of $\mathrm{Hg}$ ingested is an important consideration, especially for children and pregnant women. Studies have shown that dietary factors can affect how much $\mathrm{Hg}$ enters the body and its toxicity. MeHg enters cells through a molecular mimicry mechanism. MeHg readily binds to thiol groups. When bound to the amino acid cysteine, the $\mathrm{MeHg}-$ cysteine molecule resembles the amino acid methionine, and is able to enter cells through the large amino acid transporter 1 and 2 (LAT1 and LAT2) [12]. In worms, the amino acid transporters 1-3 are homologs to LAT1 and LAT2, which transport $\mathrm{MeHg}$ into cells [13]. If worms are fed a diet enriched in methionine, $\mathrm{MeHg}$ transport into cells is significantly decreased, as well as its toxic effects [13]. Similar effects have been observed in mammalian systems [14,15]. In addition to binding thiols, $\mathrm{MeHg}$ has high affinity for selenium. In diets enriched in selenium, $\mathrm{MeHg}$ will bind selenium and selenoproteins rather than thiols, preventing glutathione depletion and $\mathrm{MeHg}$ toxicity $[16,17]$.

The relationship between dietary fats and methylmercury exposure has gained much attention. Polyunsaturated n-3 fatty acids, such as eicosapentaenoic acid (20:5n-3, EPA) and docosahexaenoic acid (22:6n-3, DHA), have multiple health benefits, from lowering serum low-density lipoprotein levels to being cardio-protective and preventing metabolic diseases $[18,19]$. These fatty acids are high in fish species that also contain significant $\mathrm{Hg}$ levels. Therefore, understanding the relationship between PUFA and $\mathrm{Hg}$ consumption is important. Longitudinal studies performed in the Seychelle Islands have shown beneficial effects on cognition in children from maternal exposure to PUFAs found in fish contaminated with $\mathrm{MeHg}$ [20-22]. n-3 PUFAS may protect against MeHg toxicity, either by decreasing apoptosis or by reducing $\mathrm{MeHg}$ uptake [23]. Interestingly, a meta-analysis has shown that high circulating n-3 PUFA levels or fish consumption correlate with a lower risk of developing metabolic syndrome [24]. While PUFAs are an important type of fatty acid, our human diets can be varied and contain multiple other types of lipids. Little is known about the effects of other dietary lipids on $\mathrm{MeHg}$ toxicity. As high-total-fat, high-saturatedfat, and high-cholesterol diets are all implicated in the development of metabolic syndrome, 
we were interested in whether changing the bacterial strain fed to C. elegans would affect the worm's response to MeHg. We hypothesized that high-fat diets would synergize the metabolic dysfunction caused by $\mathrm{MeHg}$ exposure. To test this hypothesis, we exposed worms to $\mathrm{MeHg}$ and fed them one of four test E. coli diets: the standard diet strain (OP50), HB101 or HT115 (two diets previously shown to cause lower triglyceride accumulation than OP50), or a high-cholesterol diet (OP50 grown on plates containing twice the standard concentration of cholesterol). We then tested for $\mathrm{MeHg}$ lethality and $\mathrm{Hg}$ accumulation. We determined that the low-fat diets were more protective against $\mathrm{MeHg}$ lethality than OP50 and the high-cholesterol diet despite equivalent $\mathrm{Hg}$ accumulation. We then examined intracellular triglyceride content and lipid accumulation, as well as pro-adipogenic gene expression in worms exposed to $\mathrm{MeHg}$ and fed the test diets. As feeding in C. elegans is linked to specific neurobehavior, we assessed feeding and locomotor behaviors controlled by the dopaminergic, serotonergic, and glutamatergic neurotransmitter systems in worms exposed to $\mathrm{MeHg}$ and fed the test diets. Finally, as oxidative stress is an important determinant in neurotoxicity and metabolic toxicity, we measured reactive oxygen species (ROS) levels, protein carbonyl content, glutathione content, and antioxidant response element activation following MeHg exposure and test diet feeding.

\section{Materials and Methods}

2.1. Reagents

Unless otherwise stated, all reagents were obtained from Sigma-Aldrich (St. Louis, MO, USA). Primers used in this study included tba-1 (F: AGACCAACAAGCCGATGGAG, R: TCCAGTGCGGATCTCATCAAC), cebp-1 (F: CACTGACATGCCGAACAACG, R: AGAGAGTCTTGTCTTGCGAAGG, sbp-1 (F: GGCGGCGAAGATTGTGATTC, R: CACTGACATGCCGAACAACG), fat-6 (F: AGAGGAGAGCAAGAAGATCCCA, R: TCACGGTTTGCCATTTTGCC), and vit-2 (F: TGATGAGTCCACCAACGAGTTC, R: TTGCTCCTCGTCTCTCTCGT).

\subsection{C. elegans Strains and Worm Maintenance}

C. elegans strains were maintained at $20^{\circ} \mathrm{C}$ on Nematode Growth Medium (NGM) plates seeded with either Escherichia coli strains OP50, HT115, or HB101, as previously described [25]. Additionally, worms were maintained on a 2x cholesterol NGM plate (10 mg/mL cholesterol) seeded with OP50. C. elegans are cholesterol auxotrophs; studies have shown that above $5 \mathrm{mg} / \mathrm{mL}$ cholesterol levels are high in the nematodes [26]. For the majority of the study, wild-type N2 worms were used. We also used the VP596 (dvls19[pAF15(gst-4::GFP [green fluorescent protein]::NLS)];vsls33[dop-3::RFP (red fluorescent protein)] strain to measure antioxidant response element activity. Both strains were obtained from the Caenorhabditis Genetic Center (CGC; University of Minnesota). The bleaching method was used to harvest eggs for synchronous L1 populations, as previously described [27]. Briefly, embryos were isolated from gravid worms using a bleaching solution $(1 \% \mathrm{NaOCl}$ and $0.25 \mathrm{M} \mathrm{NaOH})$ followed by a sucrose gradient to segregate eggs from worm and bacterial debris. Synchronized L1 worms were treated with $\mathrm{MeHg}$ for $30 \mathrm{~min}$ in M9 liquid buffer at $25^{\circ} \mathrm{C}$ on a tube rotator, and then plated on the NGM plates seeded with the differing E. coli diets. We have previously shown that these concentrations are below the $\mathrm{LD}_{50}$ for $\mathrm{MeHg}$ in C. elegans and correlate to concentrations of $\mathrm{MeHg}$ in the worm that are below the US EPA reference dose of $0.1 \mu \mathrm{g} / \mathrm{kg} / \mathrm{d}[28,29]$.

\subsection{Dose-Response Survival Curves}

The lethal dose 50\% ( $\left.\mathrm{LD}_{50}\right)$ of MeHg for N2 C. elegans strains fed differing diets was determined by treating 5000 synchronized L1 worms with doses ranging from 1 to $200 \mu \mathrm{M}$ $\mathrm{MeHg}$ for $30 \mathrm{~min}$ in M9 liquid buffer at $25^{\circ} \mathrm{C}$ on a tube rotator. All exposures were carried out in triplicate and repeated 5 times. After treatment, worms were washed 3 times with M9 buffer, transferred to OP50-, HT115-, or HB101-seeded NGM plates or OP50-seeded 2x cholesterol NGM plates, and manually counted for lethality $24 \mathrm{~h}$ after MeHg treatment. 


\subsection{Mercury Quantification}

Inductively coupled mass spectrometry (ICP-MS, Agilent 8800 ICP-QQQ) was used to measure intraworm concentrations of $\mathrm{Hg}$. A total of 50,000 worms per sample were treated with $\mathrm{MeHg}$ and then fed for $48 \mathrm{~h}$ on one of the four test diets before washing with M9 and flash-freezing in liquid nitrogen. The samples were then sonicated. After centrifugation, an aliquot of the supernatant was used to measure protein concentration via the BCA assay. The rest of the sample was digested in the microwave with $1.6 \mathrm{~mL}$ bidest $\mathrm{H}_{2} \mathrm{O}, 250 \mu \mathrm{L}$ $\mathrm{HNO}_{3}$ suprapur $^{\circledR}$ and $250 \mu \mathrm{L} \mathrm{HCl}$ suprapur ${ }^{\circledR}$. Hg content was measured with No gas mode ICP-MS. Rhodium $(0.01 \mu \mathrm{g} / \mathrm{L})$ was used as internal standard. The calibration was prepared in $10 \% \mathrm{HNO}_{3}$ suprapur ${ }^{\circledR}$ and $10 \% \mathrm{HCl}$ suprapur ${ }^{\circledR}$ using a concentration range of $1-300 \mathrm{ng} / \mathrm{L}$. The washout solution contained $1 \mathrm{ppm}$ gold in $5 \% \mathrm{HNO}_{3}$ and $5 \% \mathrm{HCl}$. The content of $\mathrm{Hg}$ was calculated by dividing total $\mathrm{Hg}$ by total protein ( $\mathrm{ng} \mathrm{Hg} / \mathrm{mg}$ protein).

\subsection{Triglyceride Quantification}

The Enzychrom ${ }^{\mathrm{TM}}$ triglyceride quantification kit (BioAssay Systems, Hayward, CA, USA) was used to measure total intracellular triglycerides. Following the $\mathrm{MeHg}$ treatment, 200,000 worms were fed the test diets for $48 \mathrm{~h}$ and were homogenized in triglyceride assay buffer. Extracts were incubated for $30 \mathrm{~min}$ at room temperature with the triglyceride assay reagent mix, and absorbency (optical density: $570 \mathrm{~nm}$ ) was read. Data are expressed as mmol triglycerides $/ \mu \mathrm{g}$ protein.

\subsection{Nile Red Staining}

Previously, we have shown that fat storage sites are increased by MeHg through two methods, BODIPY 493/503 and Nile Red [10]. As the Nile Red method amends itself to screening multiple treatment groups, we chose to quantify fat storage sites using this method. Twenty thousand L1 worms were incubated with $\mathrm{MeHg}$, washed, and were transferred to OP50-, HT115-, or HB101-seeded NGM plates or OP50-seeded 2x cholesterol NGM plates. Seventy-two $h$ after treatment, worms were washed off the plates and were fixed for Nile Red staining, as previously described [30]. One thousand worms were first washed with $0.1 \%$ triton in PBS, and then fixed in $40 \%$ isopropanol for 3 min. Fixed worms were next incubated with $3 \mu \mathrm{g} / \mathrm{mL}$ Nile Red in $40 \%$ isopropanol for $30 \mathrm{~min}$ followed by another M9 wash step to remove excess dye. Worms were loaded onto a 96-well plate and Nile Red fluorescence was read at excitation $560 \mathrm{~nm}$, emission $590 \mathrm{~nm}$. Data were normalized to worm number and protein levels.

\subsection{RNA Isolation and Real-Time qPCR Gene Expression}

RNA from 20,000 worms per treatment was isolated using Trizol solution followed by chloroform extraction. cDNA was then synthesized from $1 \mathrm{mg}$ of total RNA using the Appled Biosystems' High-Capacity cDNA Reverse Transcription Kit (Thermo Fisher Scientific). Real-time PCR analysis was then performed using PerfeCTa SYBR Green FastMix (QuantaBio, Beverly, MA, USA).

\subsection{Feeding Behavioral Analysis}

L1 worms were seeded on OP50-, HT115-, or HB101-spread NGM plates, or OP50spread 2x cholesterol NGM plates following MeHg treatment and were assessed $72 \mathrm{~h}$ post-treatment for behaviors associated with nematode feeding; these include pharyngeal pumping, locomotion, and the basal slowing response. For pharyngeal pumping, 10 worms were transferred to fresh NGM/2x cholesterol NGM plates spread with the corresponding E. coli diet and the number of pharynx pumps was counted for $30 \mathrm{~s}$. Locomotion was assessed by the body-bend assay: worms were plated on an unseeded NGM/2x cholesterol NGM plate and scored for the number of forward-directed body-bends during a $30 \mathrm{~s}$ timespan. The basal slowing response assay was used to measure dopamine-dependent behavior that mediates the worm's slowing movement to consume food. The basal slowing response assay was performed as previously described [31]. The number of forward- 
directed body-bends was scored for worms placed either on NGM or 2x cholesterol NGM plates seeded or unseeded with OP50, HB101, or HT115 E. coli. For all behavioral assays, $2 x$ cholesterol plates were only used for experiments where worms were fed OP50 grown on $2 x$ cholesterol plates. These data are presented as the change in body-bends, calculated by subtracting the number of body-bends of worms plated on E. coli-seeded plates from the number of body-bends of worms plated on unseeded plates.

\subsection{Glutathione Quantification}

The 5,5'-dithiobis-2-nitrobenzoic acid-GSH disulfide reductase recycling method was used to measure total intracellular glutathione (GSH) levels, as previously described [32] in whole worm extracts from 30,000 worms.

\subsection{Intracellular Reactive Oxygen Species Determination}

Intracellular reactive oxygen species (ROS) were measured using 2,7-dichlorodihydrofluorescein diacetate (DCFD), as previously described [33]. Briefly, 20 worms treated with $\mathrm{MeHg}$ and fed on the test diet for $72 \mathrm{~h}$ were loaded onto a black 96-well plate and treated with $25 \mu \mathrm{M}$ DCFDA. Green fluorescence (excitation $490 \mathrm{~nm}$, emission $520 \mathrm{~nm}$ ) was read immediately and subsequently every $30 \mathrm{~min}$ for $6 \mathrm{~h}$.

\subsection{Protein Oxidation Quantification}

In this study, 2,4-dinitrophenylhydrazine (DNPH) labeling was used to measure protein carbonylation, a type of protein oxidation, as previously described. Using Yasuda et al.'s method [34], 50,000 treated and test-diet-fed worms were sonicated in $5 \mathrm{mM}$ EDTA with protease inhibitors. Protein was precipitated out of solution using $20 \%$ trichloroacetic acid and then incubated with $10 \mathrm{mM}$ DNPH for $1 \mathrm{~h}$. After excess DNPH was washed off, samples were suspended in $6 \mathrm{M}$ guanidine hydrochloride, loaded onto a 96-well plate, and absorbance was read at $380 \mathrm{~nm}$. Concentration of oxidized protein was calculated using Beer-Lambert's law (molar absorptivity coefficient of DNPH is $21 \mathrm{mM}^{-1} \mathrm{~cm}^{-1}$ ). Data were normalized to total protein concentration.

\subsection{Oxidative Stress Reporter Assay}

Activation of the antioxidant response element was measured using the VP596 strain, which expresses GFP under the control of the promoter for the SKN-1 target GSH S transferase 4 (gst-4). SKN-1, the worm homolog of nuclear factor (erythroid-derived-2)like 2 (Nrf2), is a transcription factor that binds the antioxidant response element and transcribes antioxidant genes in response to environmental insults. VP596 worms also express RFP under the dop-3 promoter. L1 VP596 worms were treated with $\mathrm{MeHg}$ (10 or $20 \mu \mathrm{M}$ ) for $30 \mathrm{~min}$, washed, and transferred to agar plates to be maintained for $72 \mathrm{~h}$ on the different diets. Worms were then washed off the plates, loaded onto a 96-well plate, and levels of RFP and GFP florescence were measured (RFP: excitation $544 \mathrm{~nm}$, emission $590 \mathrm{~nm}$; and GFP: excitation $485 \mathrm{~nm}$, emission $520 \mathrm{~nm}$ ). Antioxidant response element activity was represented as GFP florescence divided by RFP florescence (normalization to worm number).

\subsection{Statistics}

Statistical analyses were performed using Prism 8 software (Graphpad, San Diego, CA, USA). Statistical analysis of significance was carried out either by Student's $t$-test of $\mathrm{LD}_{50}$ (Figure 1) or two-way analysis of variance (ANOVA) followed by Tukey's multiple comparisons test. Values of $p<0.05$ were considered statistically significant. 

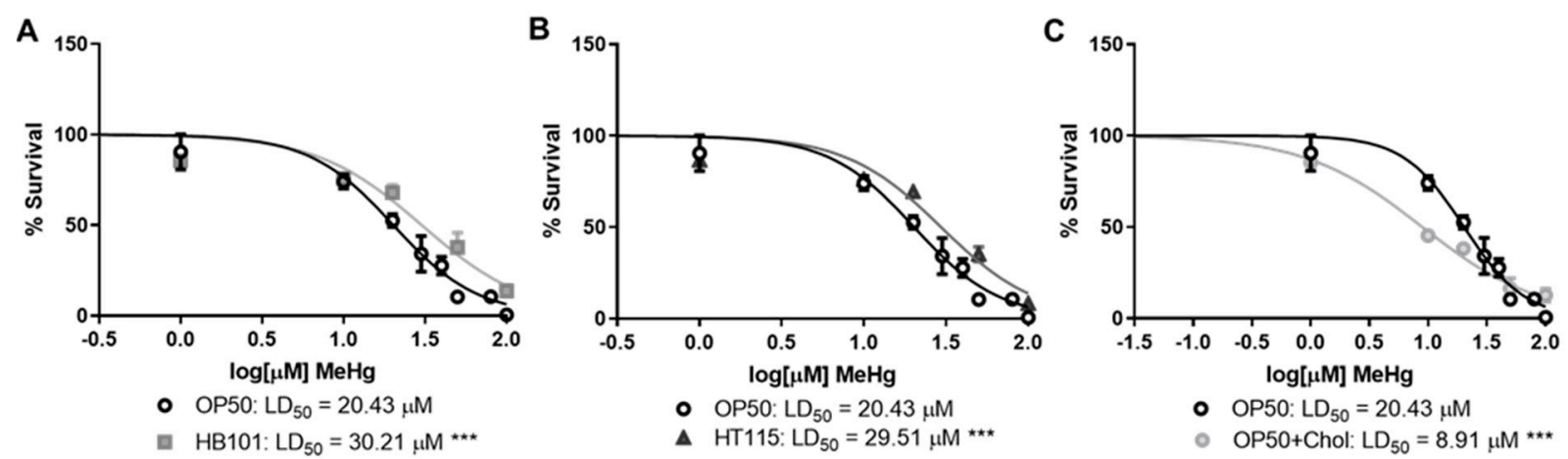

Figure 1. Diet affects MeHg toxicity. N2 worms were treated with increasing concentrations of $\mathrm{MeHg}$ for $30 \mathrm{~min}$ and then transferred to NGM plates seeded with either OP50 or (A) HB101, (B) HT115, or (C) OP50 supplemented with cholesterol. Dose-response survival curves were generated and $\mathrm{LD}_{50}$ values were calculated from five independent experiments. *** $p<0.001$ as compared with N2 MeHg-treated worms fed OP50.

\section{Results}

\subsection{Bacterial Diet Affects $\mathrm{MeHg}$ Toxicity}

As diet is a major environmental factor in health and disease development, we examined whether altering the strain of $E$. coli fed to wild-type N2 worms would affect $\mathrm{MeHg}$ toxicity. The standard E. coli diet used in nematode culture is OP50. This strain was originally selected due to its ability to form a thin, transparent monolayer, allowing for ease of visualization of the worms under a microscope [35]. How well the OP50 strain emulates the nutrition that a C. elegans worm would receive in the wild has not been accurately determined. In addition to the standard OP50 diet, we selected two diets previously shown to be lower in dietary lipids: HB101 and HT115 [36]. In comparison to OP50, worms fed HB101 had $20 \%$ fewer free fatty acids and 50\% fewer triglycerides [36]. Furthermore, the fatty acid content of triglycerides in worms fed HB101 contained $~ 50 \%$ fewer branched fatty acids and significantly increased the monounsaturated fatty acid percentage in total fatty acids than worms fed OP50 [36]. In comparison to worms fed OP50, worms fed HT115 had $~ 50 \%$ less triglycerides but did not have significant differences in total free fatty acid content or fatty acid content [36]. Similar to HB101, worms fed HT115 had $50 \%$ fewer branched-chain fatty acids in their triglycerides as compared to OP50 worms. Feeding worms either HT115 or HB101 has no effect on the mean lifespan of C. elegans, but does result in decreased basal fat storage of dietary lipids [36]. Lastly, we created a high-cholesterol diet by feeding OP50 E. coli twice the standard cholesterol concentration in NGM plates. We exposed N2 worms to increasing concentrations of MeHg, plated them on the four different diets, and generated dose-response survival curves (Figure 1). Worms fed either HB101 or HT115 were more resistant to the toxic effects of $\mathrm{MeHg}$, exhibiting a right-hand shift in their dose-response curves as compared to N2 fed OP50 $\left(\mathrm{LD}_{50} \mathrm{~s}\right.$ of 30.21 and $29.51 \mu \mathrm{M}$ for HB101 and HT115, respectively, as compared to $20.43 \mu \mathrm{M}$ for OP50). In contrast, worms fed the $2 x$ cholesterol OP50 diet were more sensitive to $\mathrm{MeHg}$, showing a left-hand shift in their dose-response curves, as compared to N2 fed OP50 (LD ${ }_{50}$ of $8.91 \mu \mathrm{M})$. These data suggest that a bacterial diet affects the toxicity of $\mathrm{MeHg}$ in nematodes.

\subsection{Diet Did Not Alter Mercury Accumulation}

Dietary components have been shown to affect the accumulation of MeHg; for example, the amino acid methionine competes with $\mathrm{MeHg}$ for passage through the large amino acid transporter into cells [12]. We therefore were interested in whether the differential toxicity to $\mathrm{MeHg}$ among the worms fed the four diets was due to differential accumulation of MeHg in the worms. N2 worms were treated with either 10 or $20 \mu \mathrm{M} \mathrm{MeHg}$ and plated for $48 \mathrm{~h}$ on NGM plates that contained one of the four test diets. Levels of $\mathrm{Hg}$ in the worms were quantified by inductively coupled mass spectrometry (ICPMS), as previously described [37]. N2 worms fed either the HB101, HT115, or 2x cholesterol OP50 diet accumu- 
lated similar levels of $\mathrm{Hg}$ following the 10 or $20 \mu \mathrm{M}$ MeHg treatments as compared to the worms fed OP50 and treated with 10 or $20 \mu \mathrm{M} \mathrm{MeHg}$ (Figure 2). Worms treated with $10 \mu \mathrm{M}$ $\mathrm{MeHg}$ and fed HT115 appeared to have lower levels of Hg accumulation than worms fed OP50 and treated with $\mathrm{MeHg}$; however, this trend was not statistically significant. This suggests that there was no difference in transport, accumulation, or elimination between the worms fed the four diets that could account for the variance in the dose-response curves seen in Figure 1.

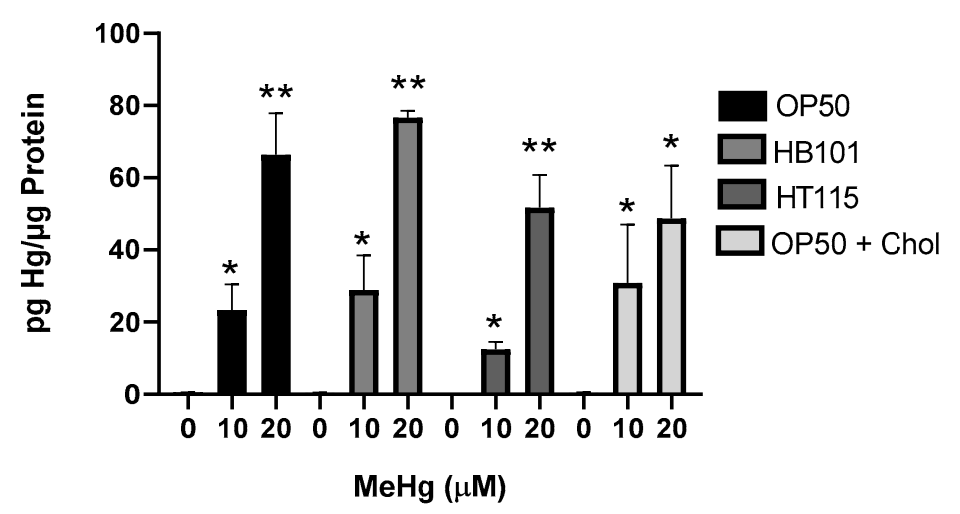

Figure 2. Bacterial diet does not affect $\mathrm{MeHg}$ content in worms. $\mathrm{Hg}$ content was measured by ICPMS in worms fed OP50, HT115, HB101, or OP50+ cholesterol diets 48 h after MeHg treatment. Hg levels are expressed as $\mathrm{pg} \mathrm{Hg} / \mu \mathrm{g}$ protein. Data represent four independent experiments. ${ }^{*} p<0.05$, ** $p<0.01$ as compared to untreated, OP50-fed control.

\subsection{Bacterial Diet Altered Lipid Accumulation in Response to $\mathrm{MeHg}$}

We have previously shown that MeHg increases the triglyceride content of N2 worms fed OP50 [10]. As HB101, HT115, and 2x cholesterol OP50 contained different lipid profiles to OP50, we were interested in whether the worms would accumulate lipids in response to MeHg at similar levels. L1 N2 were treated with 10 or $20 \mu \mathrm{M} \mathrm{MeHg}$ for $30 \mathrm{~min}$, and were allowed to feed on one of the four test diets and mature for $48 \mathrm{~h}$ before extraction of triglycerides. $\mathrm{MeHg}$ dose-dependently increased the total triglyceride content in N2 worms (Figure 3A-C). Triglyceride levels in the worms fed HB101 or HT115 were significantly lower than those of the N2 worms treated with $20 \mu \mathrm{M}$ (Figure 3A,B), while worms fed $2 \mathrm{x}$ cholesterol OP50 contained significantly more triglycerides in response to 10 and $20 \mu \mathrm{M}$ $\mathrm{MeHg}$ than the worms fed OP50 (Figure 3C).

A

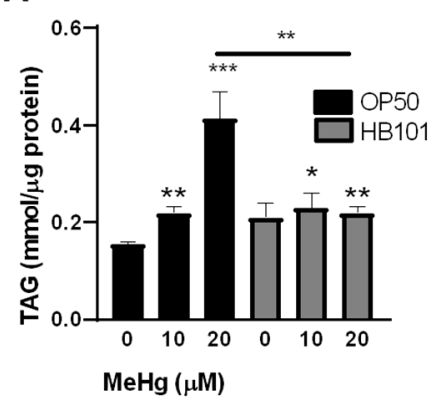

B

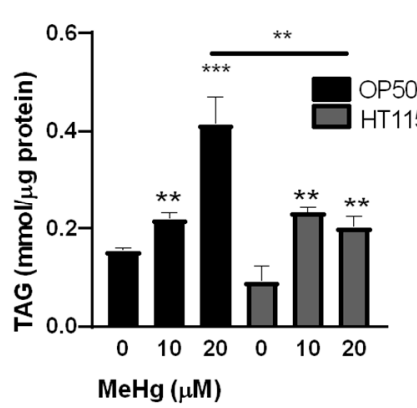

C

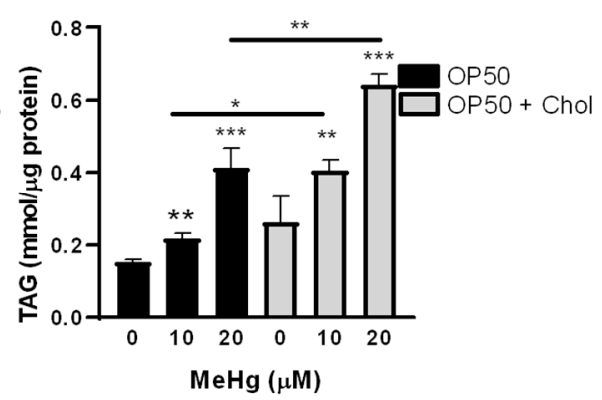

Figure 3. MeHg increases triglyceride content in worms fed a high-fat, but not low-fat, diet. Total triglycerides were measured in lysates from N2 worms fed OP50 or (A) HB101, (B) HT115, or (C) OP50 supplemented with cholesterol $48 \mathrm{~h}$ after $\mathrm{MeHg}$ treatment. Data are expressed as mean triglycerides $\mathrm{mmol} / \mu \mathrm{g}$ protein $\pm \mathrm{SEM}$. All data are representative of five independent experiments. ${ }^{*} p<0.05,{ }^{* *} p<0.01,{ }^{* * *} p<0.001$ as compared to untreated, OP50-fed control. Horizontal bars represent comparisons between Hg-treated worms fed different diets. 
We next assessed whether a bacterial diet would affect intracellular lipid storage sites. $\mathrm{N} 2$ worms were treated with $\mathrm{MeHg}$, fed one of the four test diets, and, $72 \mathrm{~h}$ after exposure, lipid storage sites were stained with Nile Red, as previously described [30]. Both 10 and $20 \mu \mathrm{M} \mathrm{MeHg}$ treatments increased lipid storage in N2 worms (Figure 4A-C). Worms that were fed either the HB101 or HT115 diets showed decreased levels of lipid storage sites in response to $\mathrm{MeHg}$ than worms fed OP50 (Figure 4A,B). However, worms fed OP50 with 2x cholesterol showed increased lipid storage sites in response to MeHg compared to worms fed OP50 with standard cholesterol levels (Figure 4C). Taken together these data suggest that lipid accumulation in response to $\mathrm{MeHg}$ is altered by the lipid content of the diet.

A

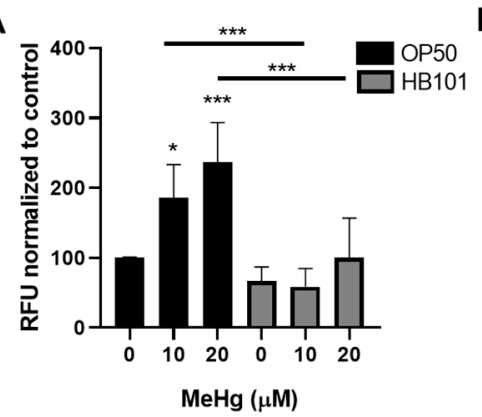

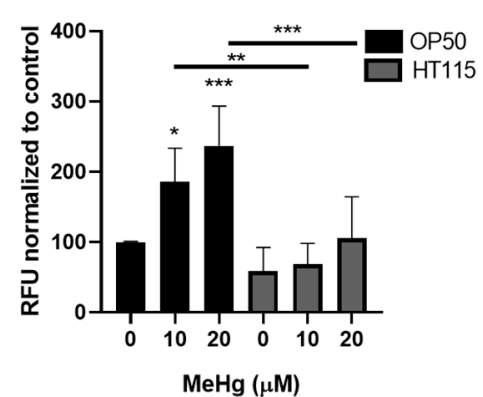

C

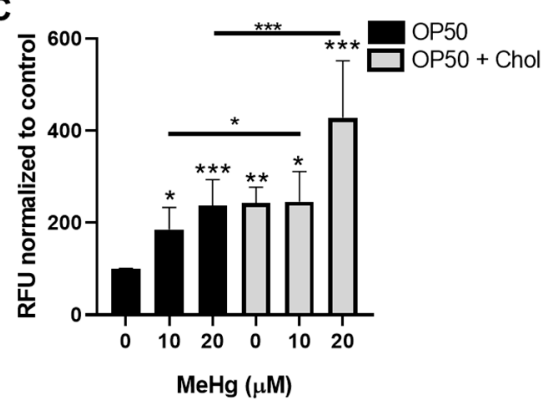

Figure 4. MeHg-induced fat accumulation in worms fed high-fat, but not low-fat, diets. Worms were treated with $\mathrm{MeHg}$ for 30 min and placed on NGM plates containing OP50 or (A) HB101, (B) HT115, or (C) OP50 supplemented with cholesterol. Then, $72 \mathrm{~h}$ after treatment, worms were fixed, stained with Nile Red, and fluorescence was measured. Data represent mean Nile Red fluorescence normalized to worm number and protein content \pm SEM from five independent experiments. ${ }^{*} p<0.05,{ }^{* *} p<0.01, * * * p<0.001$ as compared with untreated, OP50-fed control. Horizontal bars represent comparisons between Hg-treated worms fed different diets.

\subsection{MeHg-Induced Pro-Adipogenic Gene Transcription Is Diet-Dependent}

We have previously shown that $\mathrm{MeHg}$ induces the expression of several genes involved in lipid accumulation and metabolic disease [10]. MeHg increases the expression of cebp-1 (worm homolog of C/EBP) in worms fed OP50. C/EBP is a regulator of adipocyte differentiation, hyperplasia (increase in adipocyte cell numbers), and hypertrophy (adipocyte cell size) [38,39]. MeHg exposure increased the expression of cebp-1 in N2 worms fed OP50 $72 \mathrm{~h}$ post-treatment as compared to untreated controls (Figure 5A). Worms fed with either HB101 or HT115 showed significantly lower expression of cebp-1 than worms fed OP50. However, cebp-1 expression levels were not significantly different between the worms fed OP50 or OP50 with $2 x$ cholesterol, suggesting that cebp-1 induction in C. elegans is not dependent on the dietary cholesterol level.

Working in concert with C/EBP to induce adipocyte differentiation in mammals is sterol response element binding protein (SREBP, sbp-1 in worms) $[38,39]$. We have previously shown that $s b p-1$ expression is increased $24 \mathrm{~h}$ after $\mathrm{MeHg}$ treatment in worms fed OP50. Here, we report that $s b p-1$ levels in response to 10 or $20 \mu \mathrm{M} \mathrm{MeHg}$ remain elevated $72 \mathrm{~h}$ post-exposure in OP50-fed worms (Figure 5B). The expression of $s b p-1$ followed a similar trend as cebp-1 in the worms fed HT115 and HB101. These worms had significantly lower expression of $s b p-1$ in response to $\mathrm{MeHg}$ than the OP50-fed worms. In mammalian systems, the SREBP transcription factor is regulated by dietary cholesterol levels; when there is low cholesterol, the transcription factor is active; however, when there are high levels of cholesterol in cells, SREBP is degraded and the transcription factor is inactivated [40]. Similar regulatory mechanisms are present in worms. Our data show that worms fed OP50 grown on 2x cholesterol plates had no induction of the $s b p-1$ gene in response to $\mathrm{MeHg}$. 
A

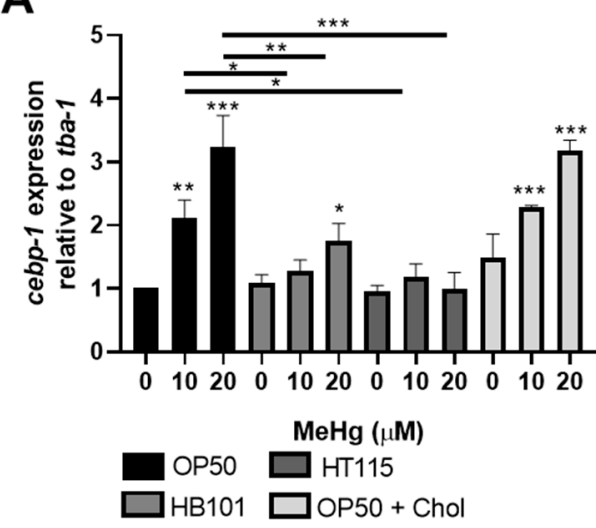

C

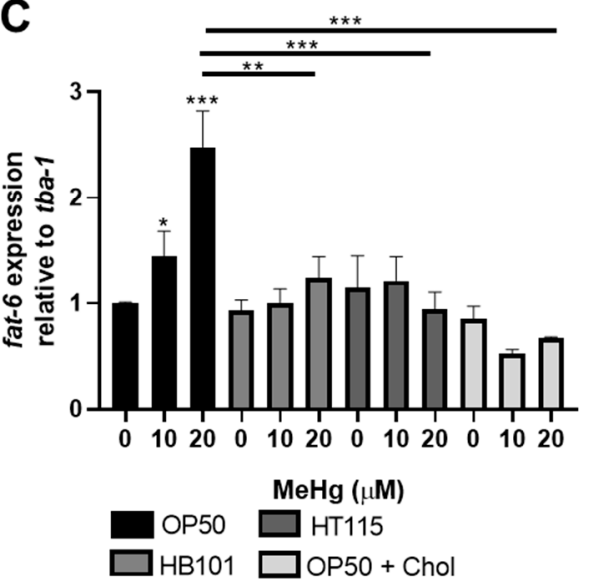

B

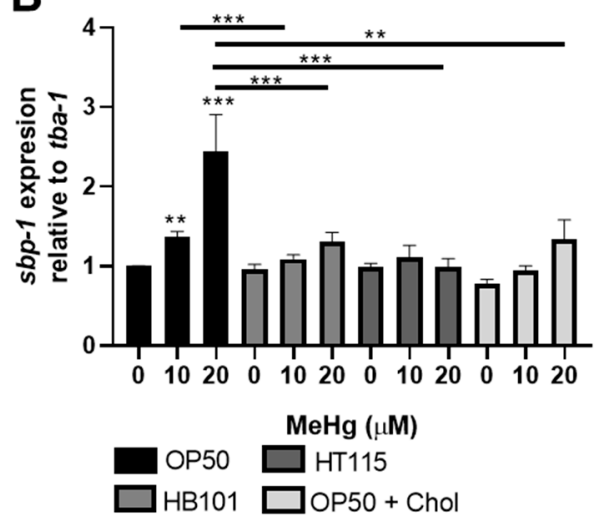

D

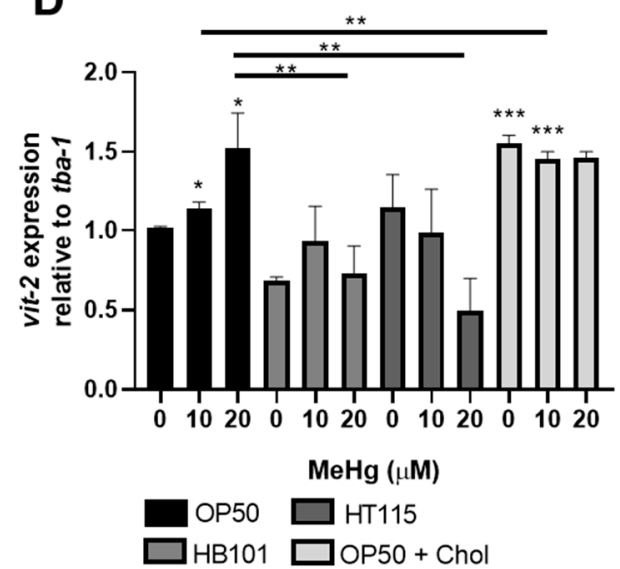

Figure 5. MeHg-induced pro-adipogenic gene expression is dependent on diet. Worms were treated with MeHg for $30 \mathrm{~min}$ and placed on NGM plates containing OP50, HB101, HT115, or OP50 supplemented with cholesterol. Then, $72 \mathrm{~h}$ after treatment, levels of (A) cebp-1 (ortholog of human C/EBP), (B) sbp-1 (ortholog of human SREBP-1), (C) fat-6 (ortholog to glycerol-3-phosphate acyltransferase), (D) vit-2 were measured by quantitative PCR and normalized to tba-1 housekeeping gene. Data are expressed as mean relative expression \pm SEM from five independent experiments. ${ }^{*} p<0.05,{ }^{* *} p<0.01,{ }^{* * *} p<0.001$ as compared to untreated OP50-fed control. Horizontal bars represent comparisons between $\mathrm{Hg}$-treated worms fed different diets.

Concurrent with the upregulation of adipogenic transcription factor $s b p-1, \mathrm{MeHg}$ increased the expression of an sbp-1-responsive gene, fat-6, the worm ortholog of stearoylCoA desaturase 1 (SCD). SCD is the rate-limiting step in the formation of monounsaturated fatty acids and triglycerides. Due to its critical roles in in obesity and insulin resistance, SCD is emerging as a potential therapeutic target for these conditions [41]. The transcription of fat- 6 is controlled by multiple transcription factors shown to be affected by $\mathrm{MeHg}$, including $n h r-49$ and $s b p-1[42,43]$. As MeHg increases sbp-1 expression, it is expected that $s b p-1$ target genes, such as fat- 6 expression, would also change. Worms treated with $\mathrm{MeHg}$ and fed OP50 had increased expression of sbp-1 (Figure 5B) and increased expression of fat-6 (Figure 5C). Likewise, worms fed HB101 or HT115 after MeHg treatment had decreased sbp-1 and fat-6 expression as compared to OP50-fed worms treated with MeHg. Furthermore, worms fed OP50 raised on NGM plates containing $2 \mathrm{x}$ cholesterol did not display sbp-1 expression or fat- 6 expression, further confirming the relationship of cholesterol levels and $s b p-1$ activity in response to $\mathrm{MeHg}$ in worms.

Finally, we examined whether diet affected lipid transport proteins in response to $\mathrm{MeHg}$ in C. elegans. Vitellogenins (vit-1-vit-6) are yolk proteins with homology to human 
apolipoprotein B-100 [44] that deliver cholesterol to oocytes through a receptor-mediated endocytosis mechanism mediated by RME-2, a member of the LDL receptor superfamily. We have previously shown that the levels of vit-2 were increased by $\mathrm{MeHg}$ treatment $24 \mathrm{~h}$ post-exposure in N2 worms fed OP50 [10]. In Figure 5D, vit-2 is increased by $\mathrm{MeHg}$ $72 \mathrm{~h}$ post-exposure in OP50-fed worms. In worms fed HB101 or HT115, MeHg did not induce vit-2 expression. Lastly, worms fed OP50 grown on 2x cholesterol NGM plates had increased expression of vit-2 compared to MeHg-treated worms fed OP50 grown on standard NGM plates. Overall, these data suggest that the different diets affected lipid accumulation in the worms in response to $\mathrm{MeHg}$, which were accommodated by a compensatory modulation of lipid binding and transport proteins.

\subsection{Feeding Behavior in Response to MeHg Is Dependent on Diet}

Feeding in C. elegans is linked to specific neuronal activity. We previously showed that $\mathrm{MeHg}$, a known neurotoxin, increased feeding in worms as well as decreased locomotion and dopaminergic behavior [10]. As we noted differences in fat accumulation between worms fed the four test diets, we were interested in whether there were differences in feeding behaviors. Worms were treated with $\mathrm{MeHg}$ and fed for $72 \mathrm{~h}$ with either OP50, HT115, or HB101 spread on NGM plates or OP50 spread on 2x cholesterol NGM plates prior to behavioral analyses. Food consumption was measured by the pharynx pump assay. $\mathrm{MeHg}$ increased food consumption in worms fed OP50 seeded on standard NGM plates (Figure 6). MeHg did not increase the rate of feeding of worms fed the HB101 or HT115 diets (Figure 6A,B). There was no statistically significant effect of NGM plate cholesterol content on MeHg-induced food consumption; worms fed OP50 on either the standard or $2 x$ cholesterol NGM plates had the same increase in pharynx pumps in response to $\mathrm{MeHg}$ (Figure 6C).
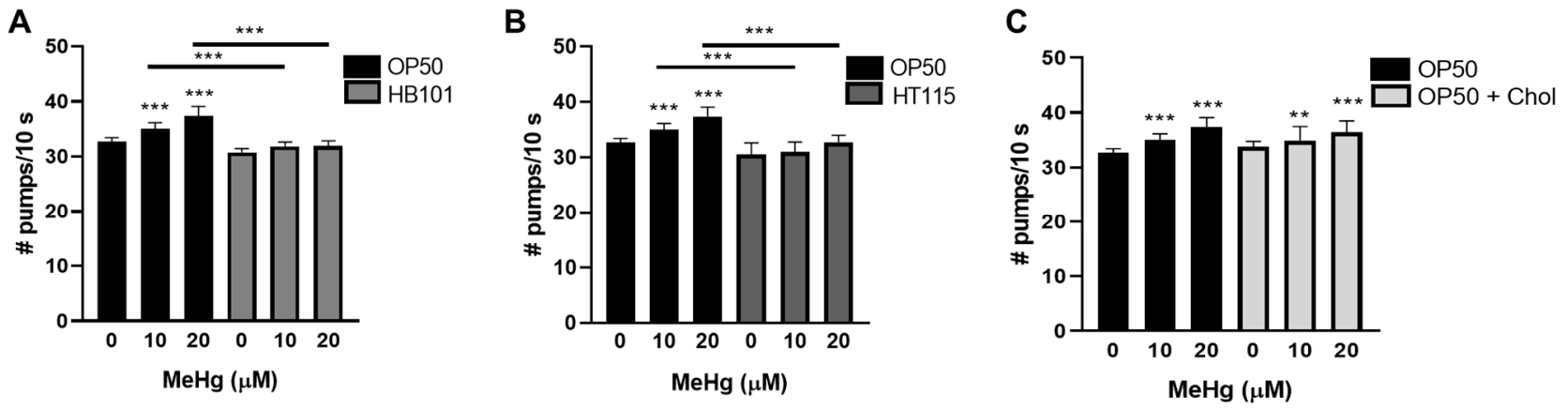

Figure 6. MeHg increases feeding behavior in worms fed a high-fat diet, but not a low-fat diet. Worms were treated with $\mathrm{MeHg}$ for 30 min and placed on NGM plates containing OP50 or (A) HB101, (B) HT115, or (C) OP50 supplemented with cholesterol. Then, $72 \mathrm{~h}$ after treatment, food consumption was analyzed by the pharynx pump assay. Data are expressed as means \pm SEM from nine independent experiments. ${ }^{* *} p<0.01,{ }^{* * *} p<0.001$ as compared with untreated, OP50-fed control. Horizontal bars represent comparisons between $\mathrm{Hg}$-treated worms fed different diets.

Locomotion and dopaminergic-dependent behavior were also investigated in $\mathrm{MeHg}$ treated worms $72 \mathrm{~h}$ following feeding on the four test diets. Previously, we showed that $\mathrm{MeHg}$ decreases locomotion rates by measuring forward-directed body-bends [10]. In comparing the four test diets, there was no significant difference in the rates of locomotion in response to $\mathrm{MeHg}$ treatment in worms fed either HB101, HT115, OP50, or OP50 on $2 x$ cholesterol NGM plates (Figure 7A). We next measured the change in body-bends on bacteria vs. off bacteria (basal slowing rate, BSR). The BSR is a direct measure of dopaminergic function, as worms deficient in dopamine production (cat-2 mutants) have no difference in the rates of locomotion on NGM plates with or without a bacterial food source [31]. Healthy N2 worms with functioning dopaminergic systems slow their locomotion on NGM plates spread with bacteria as compared to NGM plates unseeded with bacteria. MeHg is known to be toxic to dopaminergic neurons in mammals and in 
C. elegans $[28,29,45,46]$; we were therefore curious as to whether changing the worm's diet might protect the dopaminergic neurons from MeHg-induced dysfunction. BSR was measured in worms treated with $\mathrm{MeHg}$ and fed one of the four test diets. MeHg decreased the BSR in worms fed either of the four test diets, and there was no statistical difference in the BSR between worms fed the HB101, HT115, or 2x cholesterol diets as compared to the standard OP50 diet (Figure 7B). This suggests that while MeHg damages the dopaminergic functioning in worms, dopaminergic behavior is not influenced by the bacterial diet strain or cholesterol level.

A

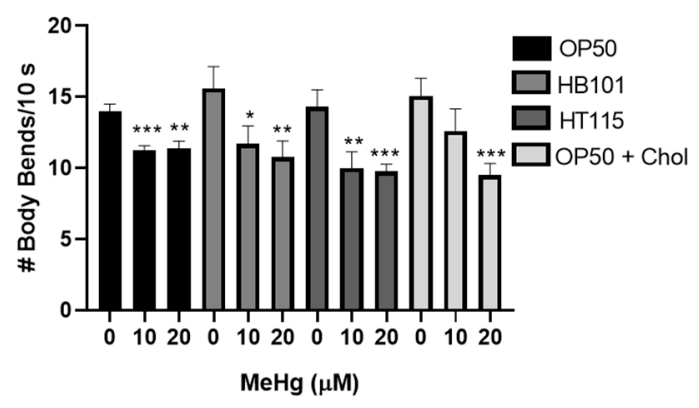

B

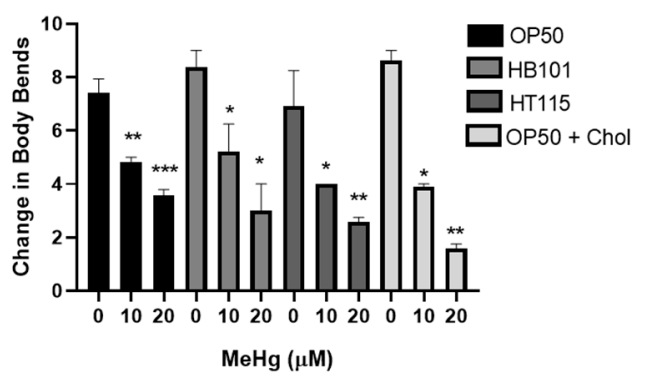

Figure 7. Locomotive and dopaminergic function in response to $\mathrm{MeHg}$ are not affected by diet. (A) Locomotion behavioral analysis was performed $72 \mathrm{~h}$ after $\mathrm{MeHg}$ treatment and feeding on the test diets. (B) Dopaminergic behavior was assessed by the basal slowing response (BSR) performed $72 \mathrm{~h}$ after MeHg treatment and feeding on the test diets. ${ }^{*} p<0.05,{ }^{* *} p<0.01$, *** $p<0.001$ as compared with untreated, OP50-fed control.

\subsection{Bacterial Diet Improves Measures of Oxidative Stress in Response to $\mathrm{MeHg}$}

Oxidative stress is a hallmark of $\mathrm{MeHg}$ exposure and can drive neurotoxicity and metabolic alterations. Dietary components can quench ROS [47-50]. We therefore investigated whether altering the bacterial diet fed to worms could prevent oxidative stress derived from $\mathrm{MeHg}$ treatment in C. elegans. Intracellular ROS were measured in worms treated with MeHg and fed either HB101, HT115, or OP50 on standard NGM plates or OP50 on 2x cholesterol NGM plates by DCFDA staining. MeHg increased intracellular ROS in worms fed OP50 on standard NGM plates (Figure 8A). Worms fed OP50 seeded on $2 x$ cholesterol plates had exacerbated ROS levels in response to MeHg. Worms fed either the HB101 or HT115 diet had significantly less MeHg-induced ROS generation. These data suggest that the dietary components in the different $E$. coli strains produced differential oxidative stress in response to MeHg. ROS damages cellular biomacromolecules, leading to lipid peroxidation and subsequent carbonyl protein adduct formation on cysteine, lysine, and histidine amino acids through Michael addition chemistry. Levels of oxidized proteins were measured using a DNPH colorimetric assay that quantified carbonyl adducts on proteins in samples derived from lysates of N2 worms treated with $20 \mu \mathrm{M} \mathrm{MeHg}$ and fed one of the four test diets. MeHg significantly increased the protein carbonyl content in worms fed OP50 seeded on standard NGM plates, which was significantly decreased in worms fed either HB101 or HT115 (Figure 8B). In contrast, worms fed OP50 seeded on 2x cholesterol NGM plates had exacerbated protein carbonyl content as compared to worms fed OP50 seeded on standard NGM plates.

We next investigated the worms' ability to mount an antioxidant response to $\mathrm{MeHg}$ following feeding with the test diets. GSH is the main intracellular thiol that is responsible for maintaining the redox environment of the cell. MeHg readily binds free thiols, such as those present on GSH. As previously observed [28], treatment with 10 or $20 \mu \mathrm{M} \mathrm{MeHg}$ led to a $20 \%$ decrease in total GSH levels in worms fed OP50 seeded on standard NGM plates (Figure 8C). Worms fed HB101 had significantly higher basal levels of GSH than worms fed OP50. MeHg treatments decreased the levels of GSH in HB101-fed worms as compared to untreated worms fed HB101; however, the levels of GSH in MeHg-treated HB101 fed worms were significantly higher than those in OP50-fed MeHg-treated worms. 
Worms fed HT115 showed a minimal decrease in GSH in response to MeHg; however, in comparing worms treated with $\mathrm{MeHg}$, the HT115-fed worms contained more GSH than the OP50-fed worms. Similar to the oxidized protein and intracellular ROS data, worms fed OP50 seeded on 2x cholesterol plates had exacerbated GSH loss in comparison to worms fed OP50 seeded on standard NGM plates.

A

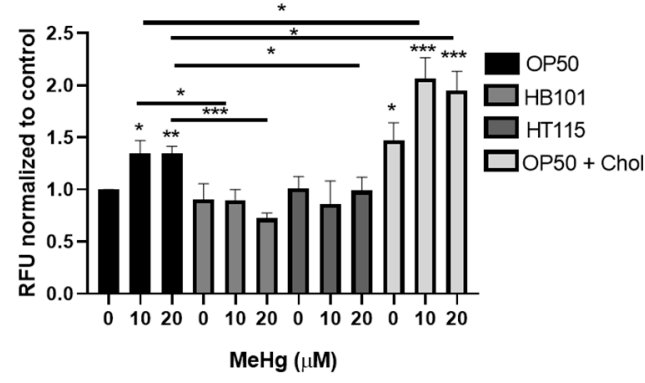

C

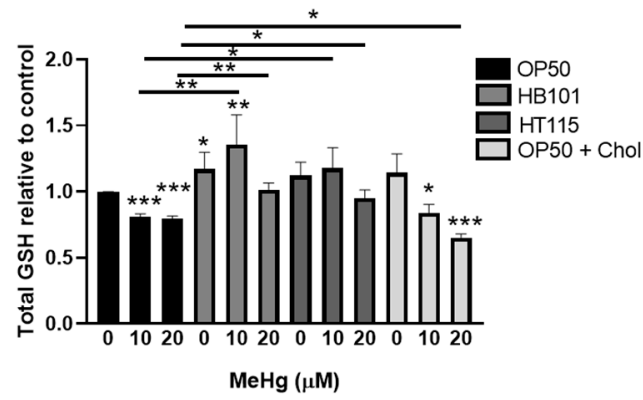

B

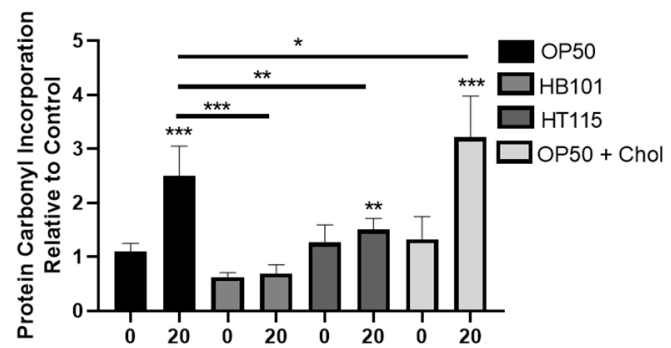

D

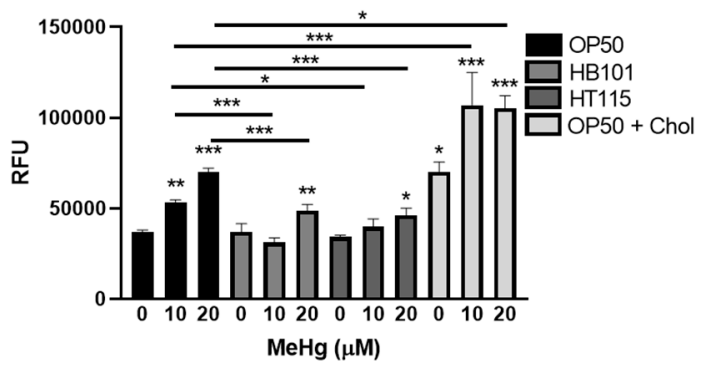

Figure 8. Diet affects MeHg-induced oxidative stress. Worms were treated with $\mathrm{MeHg}$ for $30 \mathrm{~min}$ and placed on agar plates spread with either OP50, HB101, Ht115, or OP50 supplemented with cholesterol. Measures of oxidative stress were assessed $24 \mathrm{~h}$ after MeHg treatment. (A) ROS levels were measured through DCFDA fluorescence. Data are expressed as mean fluorescence \pm SEM for 6 independent experiments (B) Protein carbonyl levels were measured and normalized to protein content. Data are expressed as means \pm SEM from 5 independent experiments. (C) Total GSH levels were measured and normalized to protein content. Data are expressed as mean \pm SEM from five independent experiments. (D) Quantification of GFP fluorescence of VP596 transgenic worms expressing GFP under the gst-4 promoter. Data are expressed as means fluorescence \pm SEM from 5 independent experiments. ${ }^{*} p<0.05,{ }^{* *} p<0.01,{ }^{* * *} p<0.001$ as compared with untreated, OP50-fed control. Horizontal bars represent comparisons between Hg-treated worms fed different diets.

Finally, we examined the effect of the different diets on the ability of the worms to generate antioxidant defense proteins. Phase II metabolic genes and antioxidant defense enzymes are regulated by the activity of the Nrf2 (Nuclear factor erythroid 2-related factor 2) (SKN-1 in C. elegans) transcription factor. MeHg is known to induce Nrf2 in cell culture and in nematodes [51,52]. We used the VP596 strain, which expresses GFP under the control of the promoter for the SKN-1 target gst-4. MeHg treatment significantly increased the amount of GFP fluorescence in worms fed OP50 seeded on standard NGM plates (Figure 8D), indicating increased SKN-1 activity. Worms fed either HB101 or HT115 had significantly less GFP fluorescence in response to $\mathrm{MeHg}$ treatment than the OP50-fed worms. This suggested that there was less oxidative stress, and therefore less SKN-1 activity, in HB101- and HT115-fed worms than in the OP50-fed worms. In contrast, worms fed OP50 seeded on 2x cholesterol NGM plates had significantly more GFP fluorescence in response to $\mathrm{MeHg}$ than worms fed OP50 seeded on standard OP50, suggesting the presence of greater oxidative stress and SKN-1 activity.

\section{Discussion}

Herein, we demonstrate for the first time that the toxicity of MeHg in C. elegans is dependent on the strain of E. coli used as a food source. MeHg is a known neurotoxic agent that has long been understood to cause neurological changes and is emerging as a 
player in metabolic diseases. C. elegans is a useful model organism to study the effects of the metabolic changes induced by MeHg because of its evolutionarily conserved fat and sugar metabolic pathways [53]. We have previously shown that $\mathrm{MeHg}$ causes metabolic alterations in C. elegans that lead to decreased nicotinamide adenine dinucleotide $\left(\mathrm{NAD}^{+}\right)$ cofactor levels, mitochondrial dysfunction, and oxidative stress [28]. We also found that $\mathrm{MeHg}$ increases the transcription of cepb-1 (ortholog to human C/EBP), nhr-49 (ortholog to human peroxisome proliferator activated receptor gamma, PPAR $\gamma$ ), and $s b p-1$ (ortholog to human sterol response element binding protein-1, SREBP-1), pro-adipogenic transcription factors implicated in MS, as well as a number of other genes involved in lipid synthesis and transport [10]. All of these findings were under the context that the worms were consuming the standard OP50 E. coli diet. It was recently shown that feeding C. elegans dehydrated dead OP50 significantly decreased the susceptibility of worms to MeHg [54]. These data suggest that the diet fed to C. elegans is an important determinant of its susceptibility to the toxic effects of $\mathrm{MeHg}$.

$\mathrm{MeHg}$ enters the human body primarily through fish consumption. For decades, studies of populations in the Seychelles, Faroe Islands, and Arctic Canada have followed cohorts of individuals exposed to $\mathrm{MeHg}$ in their diet and have produced, at times, conflicting data [55-57]. While there are multiple explanations for these discrepancies, from the types of fish eaten, to co-contaminants (such as polychlorinated biphenyls, PCBs) and/or genetic polymorphisms present in the populations, background diet composition has not been fully taken into consideration in these comparisons. Our data show in worms that the strain of E. coli can drastically alter the toxicity of $\mathrm{MeHg}$ independently of the amount of MeHg that accumulates in the worm. The strains HB101 and HT115, which are lower in triglycerides and free fatty acids than OP50 [36], conferred protection to worms from $\mathrm{MeHg}$ lethality, lower fat accumulation and adipogenic gene transcription, and decreased levels of oxidative stress than worms fed OP50. Conversely, worms fed OP50 supplemented with excess cholesterol were more susceptible to $\mathrm{MeHg}$ and had increased lipid accumulation and oxidative stress as compared to worms fed OP50 with a standard concentration of cholesterol in the NGM. Recent data have shown that $C$. elegans in the wild eat a varied diet comprising bacteria not only in the genus Escherichia, but also in the genera Sphingomonas, Xanthomonas, and Methylobacterium [58]. These diets confer differences in lifespan, development, reproduction, and gene expression [58]. It remains to be determined how feeding any of these non-E. coli species to worms might affect the toxicity of MeHg.

Our data examining lipid accumulation and $\mathrm{MeHg}$ toxicity in the context of higherand lower-fat diets in C. elegans are in agreement with previous findings in rodents and humans. KK-Ay type 2 diabetic mice, which have higher body fat than C57BL/6J mice, had lower blood clearance of $\mathrm{MeHg}$ and increased neurological damage compared to C57BL/6J mice [59,60]. Likewise, high-fat diets and $\mathrm{MeHg}$ were shown to result in similar lipid and cholesterol accumulation and steatosis in the liver [61]. In a toxicokinetic study, Rowland et al. fed mice either a standard diet or a synthetic diet that was high in protein and low in fat. The mice fed the synthetic diet accumulated less $\mathrm{Hg}$ in their body than the standard-pellet-fed mice [62]. However, it was unclear whether the effect of this synthetic diet was due to the low fat, the high protein, or to both factors. Since Hg interacts with multiple amino acids, such as cysteine, or is antagonized by methionine, the protective effect that Rowland et al. observed may have been due to the amino acid content of the synthetic diet.

$\mathrm{MeHg}$ has been shown to increase cholesterol levels in humans and rodents. MeHg has been shown to inhibit paraoxonase activity, leading to increased low-density lipoproteins (LDL) in an Inuit population that regularly eats fish [63]. Additionally, chronic exposure to MeHg in wild-type and LDL receptor knockout mice causes hypercholesterolemia [64]. Our data support the notion that a diet rich in cholesterol potentiates the toxic effects of $\mathrm{MeHg}$ on lethality and lipid accumulation. While we did not specifically measure cholesterol levels in our worms following $\mathrm{MeHg}$ exposure and feeding on the $2 \mathrm{x}$ diet, our gene expression analysis showed that high cholesterol levels were indeed achieved 
in the worms, allowing for the inhibition of $s b p-1$ transcription. SREBP1 (sbp-1 in worms) is a transcription factor that upregulates steroid and cholesterol biosynthetic genes but is inactivated and degraded under high cholesterol levels [40].

The central nervous system plays an important role in sensing nutrients and integrating hormonal signals from the gastrointestinal tract and adipocytes to regulate caloric intake and energy expenditure [65]. In humans, the hypothalamic-pituitary axis (HPA) integrates hormonal signals including leptin, insulin, ghrelin, and adiponectin. The HPA is especially vulnerable to neurotoxicants, such as $\mathrm{MeHg}$, as the blood-brain barrier is weak in certain areas, such as the arcuate of the hypothalamus, which produces neuropeptide $\mathrm{Y}$ [66]. In vitro studies of hypothalamic neuronal cell lines treated with $\mathrm{MeHg}$ show increased expression of neuropeptides pro-omiomelanocortin (Pomc) and Agouti-related peptide (Agrp), key regulators of homeostasis [67]. Just as the CNS controls feeding and energy expenditure, diet and nutrients can affect CNS function. Diet-induced obesity and high-fat diets reduce dopamine release and reuptake, leading to disruption of the satiety circuits between nucleus accumbens (NAc) dopamine terminals and projections to the hypothalamus $[68,69]$. Long-term feeding of high-fat diets in mice depletes dopamine in the NAc, which may contribute to the development of obesity [70]. Conversely, caloric restriction in rats has led to increased dopamine and serotonin levels in the striatum and increased leptin levels in the plasma [71].

The C. elegans nervous system shows simplified neuronal control of nutrient sensing. Both humans and nematodes use dopamine, glutamate, and serotonin to control foraging, locomotion, feeding, and nutrient sensing [72]. MeHg disrupts both dopamine and glutamate signaling, while little is known about $\mathrm{MeHg}^{\prime}$ effects on serotonin. C. elegans use dopamine signaling to sense food, increase turn frequency when leaving food, and for defecation $[31,73,74]$. We have previously shown that $\mathrm{MeHg}$ decreases dopamine levels and behaviors in C. elegans fed OP50, leading to decreased locomotion and deficits in sensing the presence of food [28]. In our present study, we observed that MeHg decreased dopaminergic activity in the worms independently of which bacterial strain or cholesterol concentration was presented during the feeding. This was an unexpected result. Previously, we have shown that supplementing worms with excess nicotinamide adenine nucleotide can prevent dopaminergic damage in response to $\mathrm{MeHg}$ in C. elegans [28]. Since the low-fat HB101 and HT115 diets were more protective for MeHg lethality and metabolic dysfunction, we hypothesized that there would be less dopaminergic damage. However, $\mathrm{MeHg}$ is a well-characterized dopaminergic toxicant; the lipid level of the diet presented to the worm was irrelevant. $\mathrm{MeHg}$ caused significant dopaminergic damage that was not prevented.

The physical act of feeding in nematodes is measured by the rate of pharyngeal pumping. Serotonin regulates the pharynx muscles, allowing for food to be drawn through the mouth upon muscle contraction [75]. Serotonergic neurons coordinate the action of the cholinergic MC and glutamatergic M3 motor neurons that directly synapse on pharyngeal muscle cells [76]. Glutamate is released from the M3 neurons and activates glutamategated chloride channel AVR-15 expressed on pm4 and pm5 pharyngeal muscle cells, leading to the modulation of the duration and frequency of pharyngeal pumping [76,77]. Mutants deficient in glutamate signaling lose the ability to terminate action potentials on the pm 4 and pm5 cells, resulting in a reduced pumping rate [78]. MeHg disrupts glutamate signaling, leading to an increased glutamate concentration in the synapses and neuronal excitotoxicity [79-81]. Therefore, disruption of glutamatergic signaling by $\mathrm{MeHg}$ can negatively affect the rate of pharynx pumping. Previously, we have reported increased feeding in response to $\mathrm{MeHg}$ in C. elegans fed OP50 [10]. In our present study, worms fed HB101 or HT115 showed no increase in feeding in response to MeHg. This suggests that there may have been less damage by $\mathrm{MeHg}$ to the glutamatergic or serotoniergic neurotransmitter systems in HB101- and HT115-fed worms than in worms fed OP50. Decreased feeding, as compared to OP50, may be one of the mechanisms by which the low-fat diets protected the worms from lipid dysregulation in response to $\mathrm{MeHg}$. It is important to note that, basally, different bacterial species and bacterial strains can cause 
differential pharyngeal pumping in C. elegans $[36,58]$. Both HB101 and HT115 have been shown in L4 and young adult worms to lead to significantly lower rates of pharyngeal pumping as compared to worms fed OP50 $[58,82,83]$. Our data show no difference in basal pharynx pump rate between worms fed OP50, HB101, or HT115. This may be due to our use of older worms (adults $\sim 72 \mathrm{~h}$ post L1 stage) than in previous reports. Our data also show that the cholesterol concentration in the diet did not affect the pharyngeal pumping rate basally or in response to $\mathrm{MeHg}$. Worms fed OP50 grown on plates with 2x cholesterol concentration had a dose-dependent increase in feeding similar to worms fed OP50 grown on NGM plates with the standard cholesterol concentration.

While the nervous system is a key modulator of nutrient sensing, nutrients themselves actively signal to neurons to regulate feeding behaviors. Nematodes and mammals express neuropeptides that signal to neurons in response to the presence of nutrients. For example, FLP-20 regulates glutamatergic neurons to degrade fat and induce autophagy following starvation [84]. Nutrients send either feedforward or feedback modulation to the neurons that control the pharyngeal pump rate to either increase or decrease feeding [85]. It is unknown how these pathways are affected by $\mathrm{MeHg}$ exposure.

$\mathrm{MeHg}$ exerts many of its toxic and neurotoxic effects due to the induction of oxidative stress. ROS generation following MeHg exposure can damage membrane lipids, leading to lipid peroxidation, loss of membrane integrity, and dysfunction of neuronal signaling. Likewise, oxidative stress following $\mathrm{MeHg}$ exposure can alter gene expression or directly damage enzymes and transport proteins. Dietary factors can also influence ROS levels and oxidative stress. In our study, ROS levels were increased in worms fed OP50 following $\mathrm{MeHg}$ treatment and were exacerbated when worms were fed the $2 \mathrm{x}$ cholesterol diet. This is in agreement with previous studies demonstrating that high-fat diets are linked to increased oxidative stress, leading to mitochondrial dysfunction and additional ROS production [86,87]. Oxidative stress induced by high-fat diets has been shown to be blocked by dietary factors, such as yogurt, quercetin, geraniin (polyphenol derived from Nephelium lappaceum L. fruit rind), and Terminalia arjuna extract, to name a few [47-50]. Indeed, in our study, worms fed HB101 or HT115 following MeHg exposure had significantly lower ROS production than the worms fed OP50. ROS can damage lipids, leading to lipid peroxidation, and subsequently oxidizes proteins via a process known as protein carbonylation [88]. We observed that protein carbonylation in our study was consistent with our ROS production data. Worms fed OP50 following MeHg exposure had high levels of protein carbonylation, which was exacerbated by feeding the worms the $2 x$ cholesterol diet. Conversely, worms fed the HB101 or HT115 diets following MeHg exposure had lower levels of protein carbonylation as compared to worms fed OP50. MeHg depletes cellular GSH, leading to a reduced capacity to buffer oxidative stress [89,90]. In C. elegans, GSH was significantly decreased in worms fed OP50 or the $2 x$ cholesterol diet following MeHg treatment. Worms fed the HB101 or HT115 diet did not lose intracellular GSH content following $\mathrm{MeHg}$ exposure. While this may have been the result of less oxidative stress resulting from $\mathrm{MeHg}$ in the HB101- or HT115-fed worms, the bacterial strain fed to the worms cannot be ruled out. While total protein content is not significantly different between OP50, HT115, and HB101 [36], it remains to be determined whether the thiol levels between the three strains are similar. As a final measure of oxidative stress, we measured fluorescence from a GFP reporter strain that fluoresces upon activation of the antioxidant response element. Worms fed the OP50 or 2x cholesterol diet had increased induction of the GFP reporter in response to $\mathrm{MeHg}$, suggesting high levels of antioxidant gene induction. Worms fed HB101 or HT115 following MeHg had significantly lower levels of GFP induction than worms fed OP50, corroborating that there were lower levels of oxidative stress in response to $\mathrm{MeHg}$ in worms fed either of these lower-fat bacterial strains.

\section{Conclusions}

Altogether, our study demonstrates that dietary lipid content and cholesterol content are major determinants in the response of $\mathrm{C}$. elegans to MeHg. Worms fed diets low in 
lipids had reduced triglyceride and lipid accumulation in response to $\mathrm{MeHg}$, ate less food, and experienced less oxidative stress than worms fed the standard OP50 diet that was higher in lipid content,. Conversely, worms fed a diet high in cholesterol had increased triglyceride and lipid accumulation in response to $\mathrm{MeHg}$, and experienced more oxidative stress than worms fed the standard OP50 diet. Diet did not affect certain neurotoxicities in response to $\mathrm{MeHg}$, such as dopaminergic dysfunction; however, diet did affect the rate of feeding. These data suggest that MeHg-induced lipid dysregulation and oxidative stress is influenced by dietary factors, such as triglycerides and cholesterol, leading to metabolic changes characteristic of obesity and metabolic syndrome.

Author Contributions: Conceptualization, S.W.C.; methodology, J.L.N.-C., J.B., T.S. and S.W.C.; formal analysis, J.L.N.-C., J.B. and S.W.C.; investigation, N.C., M.M., T.N., B.K., F.I., E.N., J.L.N.C., J.B., T.S. and S.W.C.; resources, J.L.N.-C., T.S. and S.W.C.; writing-original draft preparation, J.L.N.-C., J.B. and S.W.C.; writing-review and editing, S.W.C.; supervision, J.L.N.-C., J.B. and S.W.C.; project administration, S.W.C.; Funding acquisition, J.B., T.S. and S.W.C. All authors have read and agreed to the published version of the manuscript.

Funding: This research was funded by the Husson University School of Pharmacy Research Grant, Husson University Research Fund Program, DFG Research Unit TraceAge (FOR 2558).

Institutional Review Board Statement: Not applicable.

Informed Consent Statement: Not applicable.

Data Availability Statement: Not applicable.

Conflicts of Interest: The authors declare no conflict of interest.

\section{References}

1. Chamorro-Garcia, R.; Blumberg, B. Current Research Approaches and Challenges in the Obesogen Field. Front. Endocrinol. 2019, 10, 167. [CrossRef] [PubMed]

2. Kassotis, C.D.; Stapleton, H.M. Endocrine-Mediated Mechanisms of Metabolic Disruption and New Approaches to Examine the Public Health Threat. Front. Endocrinol. 2019, 10, 39. [CrossRef]

3. Clarkson, T.W.; Magos, L. The Toxicology of Mercury and Its Chemical Compounds. Crit. Rev. Toxicol. 2006, 36, 609-662. [CrossRef] [PubMed]

4. Landrigan, P.J.; Sonawane, B.; Butler, R.N.; Trasande, L.; Callan, R.; Droller, D. Early Environmental Origins of Neurodegenerative Disease in Later Life. Environ. Health Perspect. 2005, 113, 1230-1233. [CrossRef] [PubMed]

5. Bulka, C.M.; Persky, V.W.; Daviglus, M.L.; Durazo-Arvizu, R.A.; Argos, M. Multiple metal exposures and metabolic syndrome: A cross-sectional analysis of the National Health and Nutrition Examination Survey 2011-2014. Environ. Res. 2019, 168, 397-405. [CrossRef] [PubMed]

6. Wang, X.; Mukherjee, B.; Park, S.K. Associations of cumulative exposure to heavy metal mixtures with obesity and its comorbidities among U.S. adults in NHANES 2003-2014. Environ. Int. 2018, 121, 683-694. [CrossRef]

7. Lee, K. Blood mercury concentration in relation to metabolic and weight phenotypes using the KNHANES 2011-2013 data. Int. Arch. Occup. Environ. Health 2018, 91, 185-193. [CrossRef] [PubMed]

8. Park, J.S.; Ha, K.H.; He, K.; Kim, D.J. Association between Blood Mercury Level and Visceral Adiposity in Adults. Diabetes Metab. J. 2017, 41, 113-120. [CrossRef] [PubMed]

9. Park, K.; Seo, E. Association between Toenail Mercury and Metabolic Syndrome is Modified by Selenium. Nutrients 2016, 8, 424. [CrossRef]

10. Caito, S.W.; Newell-Caito, J.; Martell, M.; Crawford, N.; Aschner, M. Methylmercury Induces Metabolic Alterations in Caenorhabditis elegans: Role for C/EBP Transcription Factor. Toxicol. Sci. 2020, 174, 112-123. [CrossRef] [PubMed]

11. Chen, D.Y.; Williams, V.J. Marine fish food in the United States and methylmercury risk. Int. J. Environ. Health Res. 2009, 19, 109-124. [CrossRef] [PubMed]

12. Simmons-Willis, T.A.; Koh, A.S.; Clarkson, T.W.; Ballatori, N. Transport of a neurotoxicant by molecular mimicry: The methylmercury-1-cysteine complex is a substrate for human L-type large neutral amino acid transporter (LAT) 1 and LAT2. Biochem. J. 2002, 367, 239-246. [CrossRef]

13. Caito, S.W.; Zhang, Y.; Aschner, M. Involvement of AAT transporters in methylmercury toxicity in Caenorhabditis elegans. Biochem. Biophys. Res. Commun. 2013, 435, 546-550. [CrossRef] [PubMed]

14. Adachi, T.; Yasutake, A.; Hirayama, K. Influence of dietary levels of protein and sulfur amino acids on the fate of methylmercury in mice. Toxicology 1994, 93, 225-234. [CrossRef]

15. Meydani, M.; Meydani, S.N.; Hathcock, J.N. Effects of dietary methionine, methylmercury, and atrazine on ex-vivo synthesis of prostaglandin E1 and thromboxane B2. Prostaglandins Leukot. Med. 1984, 14, 267-278. [CrossRef] 
16. Cabañero, A.I.; Madrid, A.Y.; Cámara, C. Selenium Long-Term Administration and Its Effect on Mercury Toxicity. J. Agric. Food Chem. 2006, 54, 4461-4468. [CrossRef] [PubMed]

17. Folven, K.I.; Glover, C.N.; Malde, M.K.; Lundebye, A.-K. Does selenium modify neurobehavioural impacts of developmental methylmercury exposure in mice? Environ. Toxicol. Pharmacol. 2009, 28, 111-119. [CrossRef]

18. Wu, H.; Xu, L.; Ballantyne, C.M. Dietary and Pharmacological Fatty Acids and Cardiovascular Health. J. Clin. Endocrinol. Metab. 2020, 105, 1030-1045. [CrossRef]

19. Djuricic, I.; Calder, P. Beneficial Outcomes of Omega-6 and Omega-3 Polyunsaturated Fatty Acids on Human Health: An Update for 2021. Nutrients 2021, 13, 2421. [CrossRef] [PubMed]

20. Strain, J.J.; Davidson, P.W.; Thurston, S.W.; Harrington, D.; Mulhern, M.S.; McAfee, A.J.; van Wijngaarden, E.; Shamlaye, C.F.; Henderson, J.; Watson, G.; et al. Maternal PUFA Status but Not Prenatal Methylmercury Exposure Is Associated with Children's Language Functions at Age Five Years in the Seychelles. J. Nutr. 2012, 142, 1943-1949. [CrossRef]

21. Strain, J.J.; Yeates, A.J.; van Wijngaarden, E.; Thurston, S.W.; Mulhern, M.S.; McSorley, E.M.; Watson, G.E.; Love, T.M.; Smith, T.H.; Yost, K.; et al. Prenatal exposure to methyl mercury from fish consumption and polyunsaturated fatty acids: Associations with child development at 20 mo of age in an observational study in the Republic of Seychelles. Am. J. Clin. Nutr. 2015, 101, 530-537. [CrossRef]

22. Strain, J.J.; Love, T.M.; Yeates, A.J.; Weller, D.; Mulhern, M.S.; McSorley, E.M.; Thurston, S.W.; Watson, G.E.; Mruzek, D.; Broberg, K.; et al. Associations of prenatal methylmercury exposure and maternal polyunsaturated fatty acid status with neurodevelopmental outcomes at 7 years of age: Results from the Seychelles Child Development Study Nutrition Cohort 2. Am. J. Clin. Nutr. 2021, 113, 304-313. [CrossRef]

23. Nøstbakken, O.J.; Bredal, I.L.; Olsvik, P.A.; Huang, T.S.; Torstensen, B.E. Effect of Marine Omega 3 Fatty Acids on MethylmercuryInduced Toxicity in Fish and Mammalian CellsIn Vitro. J. Biomed. Biotechnol. 2012, 2012, 1-13. [CrossRef] [PubMed]

24. Guo, X.-F.; Li, X.; Shi, M.; Li, D. n-3 Polyunsaturated Fatty Acids and Metabolic Syndrome Risk: A Meta-Analysis. Nutrients 2017, 9, 703. [CrossRef]

25. Brenner, S. The genetics of Caenorhabditis elegans. Genetics 1974, 77, 71-94. [CrossRef] [PubMed]

26. Cheong, M.C.; Lee, H.-J.; Na, K.; Joo, H.-J.; Avery, L.; You, Y.; Paik, Y.-K. NSBP-1 mediates the effects of cholesterol on insulin/IGF-1 signaling in Caenorhabditis elegans. Cell. Mol. Life Sci. 2013, 70, 1623-1636. [CrossRef]

27. Stiernagle, T. Maintenance of C. elegans. In C. Elegans: A Practical Approach; Hope, I.A., Ed.; Oxford University Press: New York, NY, USA, 1999.

28. Caito, S.W.; Aschner, M. NAD+Supplementation Attenuates Methylmercury Dopaminergic and Mitochondrial Toxicity in Caenorhabditis elegans. Toxicol. Sci. 2016, 151, 139-149. [CrossRef]

29. Martinez-Finley, E.J.; Chakraborty, S.; Slaughter, J.C.; Aschner, M. Early-Life Exposure to Methylmercury in Wildtype and pdr-1/parkin Knockout, C. elegans. Neurochem. Res. 2013, 38, 1543-1552. [CrossRef] [PubMed]

30. Pino, E.C.; Webster, C.M.; Carr, C.E.; Soukas, A.A. Biochemical and High Throughput Microscopic Assessment of Fat Mass in Caenorhabditis elegans. J. Vis. Exp. 2013, 73, e50180. [CrossRef]

31. Sawin, E.R.; Ranganathan, R.; Horvitz, H. C. elegans Locomotory Rate Is Modulated by the Environment through a Dopaminergic Pathway and by Experience through a Serotonergic Pathway. Neuron 2000, 26, 619-631. [CrossRef]

32. Caito, S.W.; Aschner, M. Quantification of Glutathione in Caenorhabditis elegans. Curr. Protoc. Toxicol. 2015, 64, 6.18.1-6.18.6. [CrossRef]

33. Yoon, D.S.; Lee, M.H.; Cha, D.S. Measurement of Intracellular ROS in Caenorhabditis elegans Using 2',7'-Dichlorodihydrofluorescein Diacetate. Bio-Protocol 2018, 8, e2774. [CrossRef] [PubMed]

34. Yasuda, K.; Adachi, H.; Fujiwara, Y.; Ishii, N. Protein carbonyl accumulation in aging dauer formation-defective (daf) mutants of Caenorhabditis elegans. J. Gerontol. Ser. A Boil. Sci. Med. Sci. 1999, 54, B47-B51. [CrossRef]

35. Stiernagle, T. Maintenance of C. Elegans. WormBook 2006, 11, 1-11. [CrossRef] [PubMed]

36. Brooks, K.K.; Liang, B.; Watts, J.L. The Influence of Bacterial Diet on Fat Storage in C. elegans. PLoS ONE 2009, 4, e7545. [CrossRef] [PubMed]

37. Ruszkiewicz, J.A.; de Macedo, G.T.; Miranda-Vizuete, A.; Bowman, A.B.; Bornhorst, J.; Schwerdtle, T.; Soares, F.A.A.; Aschner, M. Sex-Specific Response of Caenorhabditis elegans to Methylmercury Toxicity. Neurotox. Res. 2019, 35, 208-216. [CrossRef] [PubMed]

38. Lee, J.-E.; Schmidt, H.; Lai, B.; Ge, K. Transcriptional and Epigenomic Regulation of Adipogenesis. Mol. Cell. Biol. 2019, 39, e00601-18. [CrossRef]

39. Tang, Q.Q.; Otto, T.C.; Lane, M.D. CCAAT/enhancer-binding protein beta is required for mitotic clonal expansion during adipogenesis. Proc. Natl. Acad. Sci. USA 2003, 100, 850-855. [CrossRef]

40. Ye, J.; DeBose-Boyd, R.A. Regulation of Cholesterol and Fatty Acid Synthesis. Cold Spring Harb. Perspect. Biol. 2011, 3, a004754. [CrossRef] [PubMed]

41. Sampath, H.; Ntambi, J.M. The role of stearoyl-CoA desaturase in obesity, insulin resistance, and inflammation. Ann. N. Y. Acad. Sci. 2011, 1243, 47-53. [CrossRef] [PubMed]

42. Van Gilst, M.R.; Hadjivassiliou, H.; Jolly, A.; Yamamoto, K.R. Nuclear Hormone Receptor NHR-49 Controls Fat Consumption and Fatty Acid Composition in C. elegans. PLoS Biol. 2005, 3, e53. [CrossRef] [PubMed]

43. Nomura, T.; Horikawa, M.; Shimamura, S.; Hashimoto, T.; Sakamoto, K. Fat accumulation in Caenorhabditis elegans is mediated by SREBP homolog SBP-1. Genes Nutr. 2010, 5, 17-27. [CrossRef] [PubMed] 
44. Baker, M. Is vitellogenin an ancestor of apolipoprotein B-100 of human low-density lipoprotein and human lipoprotein lipase? Biochem. J. 1988, 255, 1057-1060. [CrossRef] [PubMed]

45. Dreiem, A.; Shan, M.; Okoniewski, R.J.; Sanchez-Morrissey, S.; Seegal, R.F. Methylmercury inhibits dopaminergic function in rat pup synaptosomes in an age-dependent manner. Neurotoxicology Teratol. 2009, 31, 312-317. [CrossRef]

46. Shao, Y.; Chan, H.M. Effects of methylmercury on dopamine release in MN9D neuronal cells. Toxicol. Mech. Methods 2015, 25, 637-644. [CrossRef]

47. Kanthe, P.S.; Patil, B.S.; Das, K.K. Terminalia arjuna supplementation ameliorates high fat diet-induced oxidative stress in nephrotoxic rats. J. Basic Clin. Physiol. Pharmacol. 2021. [CrossRef] [PubMed]

48. Chung, A.P.; Gurtu, S.; Chakravarthi, S.; Moorthy, M.; Palanisamy, U.D. Geraniin Protects High-Fat Diet-Induced Oxidative Stress in Sprague Dawley Rats. Front. Nutr. 2018, 5, 17. [CrossRef] [PubMed]

49. Lasker, S.; Rahman, M.; Parvez, F.; Zamila, M.; Miah, P.; Nahar, K.; Kabir, F.; Sharmin, S.B.; Subhan, N.; Ahsan, G.U.; et al. High-fat diet-induced metabolic syndrome and oxidative stress in obese rats are ameliorated by yogurt supplementation. Sci. Rep. 2019, 9, 20026. [CrossRef]

50. Pei, Y.; Otieno, D.; Gu, I.; Lee, S.-O.; Parks, J.S.; Schimmel, K.; Kang, H.W. Effect of quercetin on nonshivering thermogenesis of brown adipose tissue in high-fat diet-induced obese mice. J. Nutr. Biochem. 2021, 88, 108532. [CrossRef] [PubMed]

51. Ni, M.; Li, X.; Yin, Z.; Jiang, H.; Sidoryk-Wegrzynowicz, M.; Milatovic, D.; Cai, J.; Aschner, M. Methylmercury Induces Acute Oxidative Stress, Altering Nrf2 Protein Level in Primary Microglial Cells. Toxicol. Sci. 2010, 116, 590-603. [CrossRef] [PubMed]

52. Martinez-Finley, E.J.; Caito, S.; Slaughter, J.C.; Aschner, M. The Role of skn-1 in Methylmercury-Induced Latent Dopaminergic Neurodegeneration. Neurochem. Res. 2013, 38, 2650-2660. [CrossRef] [PubMed]

53. Jones, K.T.; Ashrafi, K. Caenorhabditis elegans as an emerging model for studying the basic biology of obesity. Dis. Model. Mech. 2009, 2, 224-229. [CrossRef] [PubMed]

54. Ke, T.; Aschner, M. Bacteria affect Caenorhabditis elegans responses to MeHg toxicity. NeuroToxicology 2019, 75, 129-135. [CrossRef] [PubMed]

55. Davidson, P.; van Wijngaarden, E.; Shamlaye, C.; Strain, J.; Myers, G. Putting findings from the Seychelles Child Development Study into perspective: The importance of a historical special issue of the Seychelles Medical and Dental Journal. NeuroToxicology 2020, 76, 111-113. [CrossRef] [PubMed]

56. Rice, D.C. Identification of functional domains affected by developmental exposure to methylmercury: Faroe islands and related studies. NeuroToxicology 2000, 21, 1039-1044. [PubMed]

57. Bélanger, M.-C.; Dewailly, É.; Berthiaume, L.; Noël, M.; Bergeron, J.; Mirault, M.-É.; Julien, P. Dietary contaminants and oxidative stress in Inuit of Nunavik. Metabolism 2006, 55, 989-995. [CrossRef] [PubMed]

58. Stuhr, N.L.; Curran, S.P. Bacterial diets differentially alter lifespan and healthspan trajectories in C. elegans. Commun. Biol. 2020, 3, 653. [CrossRef] [PubMed]

59. Yamamoto, M.; Motomura, E.; Yanagisawa, R.; Hoang, V.A.T.; Mogi, M.; Mori, T.; Nakamura, M.; Takeya, M.; Eto, K. Evaluation of neurobehavioral impairment in methylmercury-treated KK-Ay mice by dynamic weight-bearing test. J. Appl. Toxicol. 2019, 39, 221-230. [CrossRef]

60. Yamamoto, M.; Yanagisawa, R.; Motomura, E.; Nakamura, M.; Sakamoto, M.; Takeya, M.; Eto, K. Increased methylmercury toxicity related to obesity in diabetic KK-Ay mice. J. Appl. Toxicol. 2014, 34, 914-923. [CrossRef]

61. Leocádio, P.C.L.; Dias, R.P.; Pinto, D.V.; Reis, J.M.; Nascimento, J.C.R.; Brito, G.A.D.C.; Valença, J.T.; Foureaux, G.; Ferreira, A.J.; Windmöller, C.C.; et al. Pollutants and nutrition: Are methylmercury effects on blood pressure and lipoprotein profile comparable to high-fat diet in mice? Ecotoxicol. Environ. Saf. 2020, 204, 111036. [CrossRef] [PubMed]

62. Rowland, I.R.; Mallett, A.K.; Wise, A. The Effect of Diet on the Mammalian Gut Flora and Its Metabolic Activities. CRC Crit. Rev. Toxicol. 1985, 16, 31-103. [CrossRef] [PubMed]

63. Ayotte, P.; Carrier, A.; Ouellet, N.; Boiteau, V.; Abdous, B.; Sidi, E.A.L.; Chateau-Degat, M.-L.; Dewailly, É. Relation between Methylmercury Exposure and Plasma Paraoxonase Activity in Inuit Adults from Nunavik. Environ. Health Perspect. 2011, 119, 1077-1083. [CrossRef] [PubMed]

64. Moreira, E.; de Oliveira, J.; Dutra, M.F.; Santos, D.B.; Gonçalves, C.A.; Goldfeder, E.M.; de Bem, A.F.; Prediger, R.; Aschner, M.; Farina, M. Does Methylmercury-Induced Hypercholesterolemia Play a Causal Role in Its Neurotoxicity and Cardiovascular Disease? Toxicol. Sci. 2012, 130, 373-382. [CrossRef] [PubMed]

65. Timper, K.; Brüning, J.C. Hypothalamic circuits regulating appetite and energy homeostasis: Pathways to obesity. Dis. Model. Mech. 2017, 10, 679-689. [CrossRef] [PubMed]

66. Huang, Y.; Lin, X.; Lin, S. Neuropeptide Y and Metabolism Syndrome: An Update on Perspectives of Clinical Therapeutic Intervention Strategies. Front. Cell Dev. Biol. 2021, 9, 695623. [CrossRef] [PubMed]

67. Ferrer, B.; Peres, T.V.; Dos Santos, A.A.; Bornhorst, J.; Morcillo, P.; Gonçalves, C.L.; Aschner, M.; Vieira, T.P. Methylmercury Affects the Expression of Hypothalamic Neuropeptides That Control Body Weight in C57BL/6J Mice. Toxicol. Sci. 2018, 163, 557-568. [CrossRef] [PubMed]

68. Wallace, C.; Fordahl, S. Obesity and dietary fat influence dopamine neurotransmission: Exploring the convergence of metabolic state, physiological stress, and inflammation on dopaminergic control of food intake. Nutr. Res. Rev. 2021, 1-16. [CrossRef] [PubMed] 
69. Geiger, B.; Haburcak, M.; Avena, N.; Moyer, M.; Hoebel, B.; Pothos, E. Deficits of mesolimbic dopamine neurotransmission in rat dietary obesity. Neuroscience 2009, 159, 1193-1199. [CrossRef]

70. Estes, M.K.; Bland, J.J.; Ector, K.K.; Puppa, M.J.; Powell, D.W.; Lester, D.B. A high fat western diet attenuates phasic dopamine release. Neurosci. Lett. 2021, 756, 135952. [CrossRef] [PubMed]

71. Rojic-Becker, D.; Portero-Tresserra, M.; Martí-Nicolovius, M.; Vale-Martínez, A.; Guillazo-Blanch, G. Effects of caloric restriction on monoaminergic neurotransmission, peripheral hormones, and olfactory memory in aged rats. Behav. Brain Res. 2021, 409, 113328. [CrossRef] [PubMed]

72. Dallière, N.; Holden-Dye, L.; Dillon, J.; O'Connor, V.; Walker, R.J. Caenorhabditis elegans Feeding Behaviors. In Oxford Research Encyclopedia of Neuroscience; Oxford University Press (OUP): Oxford, UK, 2017.

73. Hills, T.; Brockie, P.J.; Maricq, A.V. Dopamine and Glutamate Control Area-Restricted Search Behavior in Caenorhabditis elegans. J. Neurosci. 2004, 24, 1217-1225. [CrossRef]

74. Vidal-Gadea, A.; Topper, S.; Young, L.; Crisp, A.; Kressin, L.; Elbel, E.; Maples, T.; Brauner, M.; Erbguth, K.; Axelrod, A.; et al. Caenorhabditis elegans selects distinct crawling and swimming gaits via dopamine and serotonin. Proc. Natl. Acad. Sci. USA 2011, 108, 17504-17509. [CrossRef] [PubMed]

75. Horvitz, H.R.; Chalfie, M.; Trent, C.; Sulston, J.E.; Evans, P.D. Serotonin and Octopamine in the Nematode Caenorhabditis elegans. Science 1982, 216, 1012-1014. [CrossRef] [PubMed]

76. Niacaris, T.; Avery, L. Serotonin regulates repolarization of the C. elegans pharyngeal muscle. J. Exp. Biol. 2003, 206, 223-231. [CrossRef]

77. Dent, J.A.; Davis, M.; Avery, L. avr-15 encodes a chloride channel subunit that mediates inhibitory glutamatergic neurotransmission and ivermectin sensitivity in Caenorhabditis elegans. EMBO J. 1997, 16, 5867-5879. [CrossRef] [PubMed]

78. Greer, E.R.; Pérez, C.L.; van Gilst, M.R.; Lee, B.H.; Ashrafi, K. Neural and Molecular Dissection of a C. elegans Sensory Circuit that Regulates Fat and Feeding. Cell Metab. 2008, 8, 118-131. [CrossRef] [PubMed]

79. Culbreth, M.; Aschner, M. Dysregulation of Glutamate Cycling Mediates Methylmercury-Induced Neurotoxicity. Adv. Neurobiol. 2016, 13, 295-305. [CrossRef] [PubMed]

80. Farina, M.; Dahm, K.C.S.; Schwalm, F.D.; Brusque, A.M.; Frizzo, M.E.; Zeni, G.; Souza, D.; da Rocha, J.B.T. Methylmercury Increases Glutamate Release from Brain Synaptosomes and Glutamate Uptake by Cortical Slices from Suckling Rat Pups: Modulatory Effect of Ebselen. Toxicol. Sci. 2003, 73, 135-140. [CrossRef] [PubMed]

81. Mutkus, L.; Aschner, J.L.; Fitsanakis, V.A.; Aschner, M. The In Vitro Uptake of Glutamate in GLAST and GLT-1 Transfected Mutant CHO-K1 Cells Is Inhibited by Manganese. Biol. Trace Elem. Res. 2005, 107, 221-230. [CrossRef]

82. Rodríguez-Palero, M.J.; López-Díaz, A.; Marsac, R.; Gomes, J.-E.; Olmedo, M.; Artal-Sanz, M. An automated method for the analysis of food intake behaviour in Caenorhabditis elegans. Sci. Rep. 2018, 8, 221-230. [CrossRef]

83. You, Y.J.; Kim, J.; Raizen, D.M.; Avery, L. Insulin, cGMP, and TGF-beta signals regulate food intake and quiescence in C. elegans: A model for satiety. Cell Metab. 2008, 7, 249-257. [CrossRef] [PubMed]

84. Kang, C.; Avery, L. The FMRFamide Neuropeptide FLP-20 Acts as a Systemic Signal for Starvation Responses in Caenorhabditis elegans. Mol. Cells 2021, 44, 529-537. [CrossRef]

85. Liu, H.; Qin, L.-W.; Li, R.; Zhang, C.; Al-Sheikh, U.; Wu, Z.-X. Reciprocal modulation of 5-HT and octopamine regulates pumping via feedforward and feedback circuits in C. elegans. Proc. Natl. Acad. Sci. USA 2019, 116, 7107-7112. [CrossRef] [PubMed]

86. Vial, G.; Dubouchaud, H.; Couturier, K.; Cottet-Rousselle, C.; Taleux, N.; Athias, A.; Galinier, A.; Casteilla, L.; Leverve, X.M. Effects of a high-fat diet on energy metabolism and ROS production in rat liver. J. Hepatol. 2011, 54, 348-356. [CrossRef] [PubMed]

87. Tan, B.L.; Norhaizan, M.E. Effect of High-Fat Diets on Oxidative Stress, Cellular Inflammatory Response and Cognitive Function. Nutrients 2019, 11, 2579. [CrossRef]

88. Fritz, K.S.; Petersen, D.R. Exploring the Biology of Lipid Peroxidation-Derived Protein Carbonylation. Chem. Res. Toxicol. 2011, 24, 1411-1419. [CrossRef]

89. Farina, M.; Aschner, M. Glutathione antioxidant system and methylmercury-induced neurotoxicity: An intriguing interplay. Biochim. Biophys. Acta (BBA)-Gen. Subj. 2019, 1863, 129285. [CrossRef] [PubMed]

90. Fujimura, M.; Usuki, F. Methylmercury-Mediated Oxidative Stress and Activation of the Cellular Protective System. Antioxidants 2020, 9, 1004. [CrossRef] [PubMed] 